\title{
GLOBAL CONFORMAL ASSOUAD DIMENSION IN THE HEISENBERG GROUP
}

\author{
JEREMY T. TYSON
}

\begin{abstract}
We study global conformal Assouad dimension in the Heisenberg group $\mathbb{H}^{n}$. For each $\alpha \in\{0\} \cup[1,2 n+2]$, there is a bounded set in $\mathbb{H}^{n}$ with Assouad dimension $\alpha$ whose Assouad dimension cannot be lowered by any quasiconformal map of $\mathbb{H}^{n}$. On the other hand, for any set $S$ in $\mathbb{H}^{n}$ with Assouad dimension strictly less than one, the infimum of the Assouad dimensions of sets $F(S)$, taken over all quasiconformal maps $F$ of $\mathbb{H}^{n}$, equals zero. We also consider dilatation-dependent bounds for quasiconformal distortion of Assouad dimension. The proofs use recent advances in self-similar fractal geometry and tilings in $\mathbb{H}^{n}$ and regularity of the Carnot-Carathéodory distance from smooth hypersurfaces.
\end{abstract}

\section{INTRODUCTION}

The conformal (Hausdorff) dimension of a metric space $(X, d)$ is

$$
C \operatorname{dim}_{H} X=\inf \operatorname{dim}_{H} Y,
$$

the infimum taken over all metric spaces $\left(Y, d^{\prime}\right)$ which are quasisymmetrically equivalent to $X$. Here $\operatorname{dim}_{H}$ denotes Hausdorff dimension. Conformal dimension was introduced by Pansu [40] in connection with the quasi-isometric classification problem in geometric group theory, and has since found significant application in uniformization and rigidity questions in analysis on metric spaces and the theory of Gromov hyperbolic groups; see e.g., Bourdon [14, [13], Keith-Laakso [29], and Bonk-Kleiner [12.

Now assume that $(X, d)$ is a fixed metric space, which we regard as an ambient environment. For a given subset $S \subset X$, we define the global conformal (Hausdorff) dimension of $S$ to be

$$
G C \operatorname{dim}_{H} S=G C \operatorname{dim}_{H}^{X} S=\inf \operatorname{dim}_{H} F(S),
$$

the infimum taken over all quasisymmetric homeomorphisms $F$ of $X$ onto itself. Global conformal dimension has been considered by the author and various collaborators [45, 10, 11, and 47] in the case when the ambient is an Euclidean space, and by Balogh [4] in the case when the ambient is the first Heisenberg group equipped with a sub-Riemannian metric.

Received by the editors August 27, 2007.

2000 Mathematics Subject Classification. Primary 30C65; Secondary 28A78, 43A80.

Key words and phrases. Quasiconformal map, conformal dimension, Assouad dimension, Heisenberg group, self-affine tiling.

Research supported by NSF grant DMS 0555869.

(C)2008 American Mathematical Society Reverts to public domain 28 years from publication 
Recently, Kovalev [33] answered in the affirmative a question posed by the author on the range of conformal and global conformal dimension. More precisely (see Theorems 1.1 and 1.2 in [33]), he showed that

$$
C \operatorname{dim}_{H} X \in\{0\} \cup[1, \infty]
$$

for all metric spaces $(X, d)$, and

$$
G C \operatorname{dim}_{H}^{V} S \in\{0\} \cup[1, \operatorname{dim} V]
$$

for any $S \subset V$, where $V$ is any separable Banach space. In short, conformal Hausdorff dimension takes on no value strictly between zero and one.

Kovalev's result (1.4) relies on the linear structure of the ambient space, and admits no obvious extension to more general ambients. For example, it leaves open the study of global conformal dimensions of subsets of the sub-Riemannian Heisenberg group or more general Carnot groups. In [46], we established (1.4) in the case $V=\mathbb{R}^{n}$ for a different notion of dimension, the so-called Assouad dimension $\operatorname{dim}_{A}$. Thus we showed that global conformal Assouad dimension $G C \operatorname{dim}_{A}^{\mathbb{R}^{n}}$ (defined as in (1.2) but with $\operatorname{dim}_{A}$ replacing $\operatorname{dim}_{H}$ ) takes on no value strictly between zero and one. In this paper, we extend the results of [46] to the Heisenberg group $\mathbb{H}^{n}$. The range of $G C \operatorname{dim}_{H}^{\mathbb{H}^{n}}$ remains unknown.

Let us briefly recall the definition of the Assouad dimension. Let $(X, d)$ be a metric space. The Assouad dimension of $X$ is

$$
\operatorname{dim}_{A} X=\inf \{s \geq 0: X \text { is } s \text {-homogeneous }\},
$$

where we say that $X$ is $s$-homogeneous if the s-homogeneity constant of $X$,

$$
C_{s}(X):=\sup _{\Lambda \subset X}(\# \Lambda)\left(\frac{\min \{d(x, y): x, y \in \Lambda, x \neq y\}}{\operatorname{diam} \Lambda}\right)^{s},
$$

is finite, the supremum taken over all finite sets $\Lambda \subset X$. Here \# $\Lambda$ denotes the cardinality of $\Lambda$. We say that $\operatorname{dim}_{A} X=\infty$ if $X$ is not $s$-homogeneous for any finite s. We always have $\operatorname{dim}_{H} X \leq \operatorname{dim}_{A} X$ [35, section 3]. For any open set $U \subset \mathbb{R}^{n}$, $\operatorname{dim}_{A} U=n$.

Assouad dimension is a global construct which records the growth rate of the cardinality of uniformly separated subsets of $X$ of a given diameter. It was introduced by Assouad [3] (under a different name) in connection with bi-Lipschitz embedding problems for doubling metric spaces. Indeed, $(X, d)$ is a doubling space (e.g., a space of homogeneous type in the sense of Coifman and Weiss) if and only if $\operatorname{dim}_{A} X$ is finite; see Heinonen [25, Chapter 10] and Luukkainen [35] for further information.

By an observation of Heinonen [25, Theorem 14.16], the conformal Assouad dimension of a uniformly perfect metric space agrees with the Ahlfors regular conformal dimension, defined as in (1.1) but with the infimum taken over all Ahlfors regular metric spaces $\left(Y, d^{\prime}\right)$ quasisymmetrically equivalent with $(X, d)$. Bonk and Kleiner [12 have indicated the relevance of Ahlfors regular conformal dimension for Cannon's conjecture and the quasisymmetric uniformization problem for metric 2 -spheres.

By analogy with (1.1) and (1.2), we define the conformal Assouad dimension of a metric space $X$, resp. the global conformal Assouad dimension of a set $S \subset X$, to be

$$
C \operatorname{dim}_{A} X=\inf \operatorname{dim}_{A} Y,
$$


the infimum taken over all metric spaces $\left(Y, d^{\prime}\right)$ which are quasisymmetrically equivalent to $X$, resp.

$$
G C \operatorname{dim}_{A} S=G C \operatorname{dim}_{A}^{X} S=\inf \operatorname{dim}_{A} F(S),
$$

the infimum taken over all quasisymmetric homeomorphisms $F: X \rightarrow X$. The inequalities $C \operatorname{dim}_{H} X \leq C \operatorname{dim}_{A} X$ and $G C \operatorname{dim}_{H} S \leq G C \operatorname{dim}_{A} S$ are obvious. In [46] (see also Proposition 2.4 in this paper) we showed that $C \operatorname{dim}_{A} X \in\{0\} \cup[1, \infty]$ for every metric space $X$ and $G C \operatorname{dim}_{A}^{\mathbb{R}^{n}} S \in\{0\} \cup[1, n]$ for every $S \subset \mathbb{R}^{n}$.

We now state our main result. Let $\mathbb{H}^{n}, n \in \mathbb{N}$, be the Heisenberg group equipped with a sub-Riemannian metric of Carnot-Carathéodory type. We recall that $\mathbb{H}^{n}$ is Ahlfors regular with homogeneous dimension $Q=2 n+2$, in particular, $\operatorname{dim}_{A} \mathbb{H}^{n}=$ $\operatorname{dim}_{H} \mathbb{H}^{n}=2 n+2$.

Theorem 1.1. For any set $S \subset \mathbb{H}^{n}$,

$$
G C \operatorname{dim}_{A}^{\mathbb{H}^{n}} S \in\{0\} \cup[1,2 n+2],
$$

and each value in the set $\{0\} \cup[1,2 n+2]$ is realized as the global conformal Assouad dimension of some bounded subset of $\mathbb{H}^{n}$.

The existence of subsets of $\mathbb{H}^{n}$ with prescribed global conformal Assouad dimension in $[1,2 n+2]$ is a modification, and extension to arbitrary $n$, of Theorem 1.3 in [4, which concerns global conformal Hausdorff dimension in $\mathbb{H}^{1}$. The hard part of Theorem 1.1 is the proof that $G C \operatorname{dim}_{A}^{\mathbb{H}^{n}}$ takes on no value strictly between zero and one. The proof of this result is similar to that of its Euclidean predecessor in [46, but also incorporates elements of the theory of self-similar fractal geometry and tilings in Heisenberg groups.

We also consider dilatation-dependent estimates for the distortion of Assouad dimension.

Theorem 1.2. Let $S \subset \mathbb{H}^{n}$ be s-homogeneous for some $s<2 n+2$ and let $F$ : $\mathbb{H}^{n} \rightarrow \mathbb{H}^{n}$ be a K-quasiconformal homeomorphism. Then $F(S)$ is $s^{\prime}$-homogeneous for some $s^{\prime}=s^{\prime}(s, n, K)<2 n+2$.

Corollary 1.3. If $S \subset \mathbb{H}^{n}$ satisfies $0<\operatorname{dim}_{A} S<2 n+2$ and $F: \mathbb{H}^{n} \rightarrow \mathbb{H}^{n}$ is quasiconformal, then $0<\operatorname{dim}_{A} F(S)<2 n+2$.

Corollary 1.3 can be made quantitative but the precise statement is somewhat technical; see Theorem 4.6 .

In section 2 we review basic notions and definitions and establish the setting for the paper. Section 3 presents relevant elements of the theory of self-similar fractal geometry and tilings in $\mathbb{H}^{n}$. In section 4 we relate Assouad dimension with porosity and prove Theorem 1.2 and Corollary 1.3 .

Section 5 contains the proof of our main result, Theorem 1.1. In subsection 5.2 we show that sets $S$ of Assouad dimension strictly less than one have global conformal Assouad dimension zero. To this end, we use the connection between Assouad dimension and porosity to identify annuli which avoid $S$ at all scales and locations, and implement a Korányi-Reimann flow of quasiconformal maps which increases the conformal moduli of the separating annuli. This leads to a reduction in the Assouad dimension.

The second part of the proof of Theorem 1.1 consists in the construction of sets which are minimal for global conformal Assouad dimension in the dimension range 
$[1,2 n+2]$. The low codimension case $[2 n+1,2 n+2]$ is particularly challenging. We consider Cantor sets of smooth closed noncharacteristic hypersurfaces in $\mathbb{H}^{n}$. Lower bounds on the global conformal dimension of such sets are established in subsection 5.1 using a method from [4. In a concluding appendix, we establish the Ahlfors regularity of these examples via the regularity theory for the CC distance recently developed by Arcozzi and Ferrari [1] and [2].

\section{REVIEW OF DEFINITIONS AND BASIC RESUltS}

2.1. Assouad dimension. We recall some basic properties of the Assouad dimension. For a more comprehensive discussion, see Luukkainen [35] and Chapter 10 of 25.

The following theorem combines (6.6) and Theorem A.5 of [35] with Exercise 10.16 of 25 .

Proposition 2.1. Let $(X, d)$ be a metric space, and let $Y, Y^{\prime} \subset X$. Then

(i) (bi-Lipschitz invariance) if $d^{\prime}$ is a metric on $X$ which is bi-Lipschitz equivalent with $d$, then $\operatorname{dim}_{A}(Y, d)=\operatorname{dim}_{A}\left(Y, d^{\prime}\right)$,

(ii) (monotonicity) $\operatorname{dim}_{A} Z \leq \operatorname{dim}_{A} Y$ if $Z \subset Y$,

(iii) $\operatorname{dim}_{A} \bar{Y}=\operatorname{dim}_{A} Y$, where $\bar{Y}$ denotes the closure of $Y$,

(iv) (finite stability) $\operatorname{dim}_{A} Y \cup Y^{\prime}=\max \left\{\operatorname{dim}_{A} Y, \operatorname{dim}_{A} Y^{\prime}\right\}$,

(v) $\operatorname{dim}_{A} Y=0$ if $Y$ is finite,

(vi) if $0<\epsilon<1$ and $d^{\prime}$ denotes the snowflake metric $d^{\prime}(x, y)=d(x, y)^{\epsilon}$ on $X$, then $\operatorname{dim}_{A}\left(Y, d^{\prime}\right)=\operatorname{dim}_{A}(Y, d) / \epsilon$,

(vii) $\operatorname{dim}_{H} Y \leq \operatorname{dim}_{A} Y$,

(viii) if $Y$ carries an $\alpha$-regular measure, then $\operatorname{dim}_{A} Y=\operatorname{dim}_{H} Y=\alpha$.

We recall that a Borel measure $\mu$ on a metric space $(X, d)$ is called (Ahlfors) $Q$-regular $(Q \geq 0)$ if there exists a constant $C<\infty$ so that

$$
C^{-1} r^{Q} \leq \mu(B(x, r)) \leq C r^{Q}
$$

for every $x \in X$ and $r \in(0, \operatorname{diam} X)$. If $X$ carries a $Q$-regular measure $\mu$, then $\mu$ is comparable with the Hausdorff measure $\mathcal{H}^{Q}$ [25. Exercise 8.11]. Similarly, when $Y$ is a subset of $X$, we say that a Borel measure $\mu$ supported on $Y$ is (Ahlfors) $\alpha$-regular $(\alpha \geq 0)$ if there exists a constant $C<\infty$ so that

$$
C^{-1} r^{\alpha} \leq \mu(Y \cap B(y, r)) \leq C r^{\alpha}
$$

for every $y \in Y$ and $r \in(0, \operatorname{diam} Y)$.

Combining Proposition 2.1 (viii) with the above observations gives the following corollary.

Corollary 2.2. If $Y$ is an $\alpha$-regular subset of $(X, d)$ and $C \operatorname{dim}_{H} Y \geq \alpha$, then

$$
G C \operatorname{dim}_{A}^{X} Y=\operatorname{dim}_{A} Y=G C \operatorname{dim}_{H}^{X} Y=\operatorname{dim}_{H} Y=\alpha .
$$

We say that $X$ is Ahlfors regular if it is $Q$-regular for some $Q \geq 0$. Every Ahlfors regular space is uniformly perfect which means that there exists $c>0$ so that $c r \leq \operatorname{diam} B(x, r) \leq 2 r$ for all $x \in X$ and $r \in(0, \operatorname{diam} X)$. This follows easily from volume considerations. The Ahlfors regular conformal dimension of a metric space $(X, d)$ is $A R C \operatorname{dim} X=\inf \operatorname{dim}_{H} Y$, the infimum taken over all Ahlfors regular spaces $\left(Y, d^{\prime}\right)$ which are quasisymmetrically equivalent to $X$. By Theorem 14.16 
of 25] $A R C \operatorname{dim} X=C \operatorname{dim}_{A} X$ for each complete, uniformly perfect metric space $(X, d)$.

In the proof of Theorem 1.1 we will employ the following lemma which goes back to Bourdon [13] and Pansu [41].

Lemma 2.3 (Bourdon, Pansu). Let $X$ be a compact $\alpha$-regular metric space, and suppose that $X$ contains a family of curves $\Gamma$ which is equipped with a probability measure $\tilde{\mu}$ so that

(i) $\inf _{\gamma \in \Gamma} \operatorname{diam} \gamma>0$, and

(ii) $\sup _{x \in X} \tilde{\mu}\{\gamma \in \Gamma: \gamma \cap B(x, r) \neq \emptyset\} \leq C r^{\alpha-1}$ for all $r>0$.

Then $C \operatorname{dim}_{H} X \geq \alpha$ and hence $C \operatorname{dim}_{A} X=C \operatorname{dim}_{H} X=\alpha$.

The range of global conformal Assouad dimension in $\mathbb{R}^{n}$ was studied in 46]. In Theorem 3.1 of 46 we showed the following result 1

Proposition 2.4. For any $S \subset \mathbb{R}^{n}, G C \operatorname{dim}_{A}^{\mathbb{R}^{n}} S \in\{0\} \cup[1, n]$.

2.2. The Heisenberg group $\mathbb{H}^{n}$. Our setting is the Heisenberg group $\mathbb{H}^{n}=$ $\mathbb{C}^{n} \times \mathbb{R}=\mathbb{R}^{2 n+1}$ equipped with the non-abelian group law

$$
(z, t) \cdot\left(z^{\prime}, t^{\prime}\right)=\left(z+z^{\prime}, t+t^{\prime}+2\left\langle z, z^{\prime}\right\rangle\right),
$$

where $\left\langle z, z^{\prime}\right\rangle=\operatorname{Im}\left(\sum_{j=1}^{n} z_{j} \overline{z_{j}^{\prime}}\right)$ denotes the standard symplectic inner product in $\mathbb{C}^{n}$. We denote by $o=(0,0) \in \mathbb{C}^{n} \times \mathbb{R}$ the identity element in $\mathbb{H}^{n}$. Writing $z=\left(z_{1}, \ldots, z_{n}\right)=\left(x_{1}+i y_{1}, \ldots, x_{n}+i y_{n}\right)$ for an arbitrary point of $\mathbb{C}^{n}$, we denote by $X_{j}=\frac{\partial}{\partial x_{j}}+2 y_{j} \frac{\partial}{\partial t}$ and $Y_{j}=\frac{\partial}{\partial y_{j}}-2 x_{j} \frac{\partial}{\partial t}, j=1, \ldots, n$, a basis for the horizontal distribution $H \mathbb{H}^{n}$, which is annihilated by the contact form $\omega=d t+2 \sum_{j=1}^{n}\left(x_{j} d y_{j}-y_{j} d x_{j}\right)$. We equip the vector subspaces $H_{p} \mathbb{H}^{n}$ with a smoothly varying inner product which makes the vector fields $\left\{X_{1}, Y_{1}, \ldots, X_{n}, Y_{n}\right\}$ orthonormal, and denote by $d_{c c}$ the associated Carnot-Carathéodory (CC) metric. Then $\left(\mathbb{H}^{n}, H \mathbb{H}^{n}, d_{c c}\right)$ has the structure of a Carnot (nilpotent stratified Lie) group; indeed, the Lie algebra of $\mathbb{H}^{n}$ is step two nilpotent, with the first layer $\mathfrak{h}$ spanned by $X_{1}, Y_{1}, \ldots, X_{n}, Y_{n}$ and the second layer $\mathfrak{v}=[\mathfrak{h}, \mathfrak{h}]$ spanned by $T=\frac{\partial}{\partial t}$. The homogeneous dimension of $\mathbb{H}^{n}$ is $2 n+2$; this is the Hausdorff dimension of $\left(\mathbb{H}^{n}, d_{c c}\right)$. The Haar measure in $\mathbb{H}^{n}$ coincides with the Lebesgue measure in the underlying Euclidean space $\mathbb{R}^{2 n+1}$ and (up to a constant multiple) with the $(2 n+2)$-dimensional CC Hausdorff measure. We denote by $\delta_{r}$ the anisotropic dilation $\delta_{r}(z, t)=\left(r z, r^{2} t\right)$ with scale factor $r>0$, by $R_{A}(z, t)=(A z, t)$ the rotation by $A \in U(n)$, and by $\pi: \mathbb{H}^{n} \rightarrow \mathbb{C}^{n}$ the projection $\pi(z, t)=z$.

For $z$ as above, we write $|z|_{\infty}=\max \left\{\left|x_{1}\right|,\left|y_{1}\right|, \ldots,\left|x_{n}\right|,\left|y_{n}\right|\right\}$ and record the elementary inequality

$$
\left|\left\langle z, z^{\prime}\right\rangle\right| \leq 2 n|z|_{\infty}\left|z^{\prime}\right|_{\infty}, \quad z, z^{\prime} \in \mathbb{C}^{n}
$$

In place of the Carnot-Carathéodory metric $d_{c c}$ we will use more computationally friendly metrics such as the gauge metric $d(p, q)=\left\|p^{-1} \cdot q\right\|$, where

$$
\|p\|=\max \left\{|z|_{\infty}, \frac{|t|^{1 / 2}}{\sqrt{2 n}}\right\}, \quad p=(z, t),
$$

\footnotetext{
${ }^{1}$ In 46 . Theorem 3.1] we assumed $S$ bounded. The extension to arbitrary $S$ is easily achieved using the invariance of Assouad dimension under conformal inversion; see [35 Theorem A.10(1)] for this result, and Proposition 2.6 for the Heisenberg analog. Compare also the last paragraph in the proof of Proposition 5.2
} 
and the related Korányi metric $d_{\mathbb{H}}(p, q)=\left\|p^{-1} \cdot q\right\|_{\mathbb{H}}$, where

$$
\|p\|_{\mathbb{H}}=\left(|z|^{4}+|t|^{2}\right)^{1 / 4}, \quad p=(z, t) .
$$

The fact that $d_{\mathbb{H}}$ is a metric is well known; that $d$ is a metric is an easy consequence of (2.1).

All three metrics, $d_{c c}, d$ and $d_{\mathbb{H}}$, are left invariant and evidently homogeneous of order one with respect to the dilations $\delta_{r}$, hence bi-Lipschitz equivalent. We denote by $B(p, r)$, resp. $B_{\mathbb{H}}(p, r)$, resp. $B_{c c}(p, r)$ the ball with center $p$ and radius $r$ in the metric $d$, resp. $d_{\mathbb{H}}$, resp. $d_{c c}$. Diameters of sets and distances between sets will always be computed in the gauge metric and denoted diam, dist. We write $\operatorname{dim}_{H} S$, resp. $\operatorname{dim}_{A} S$, for the Hausdorff, resp. Assouad, dimension of a set $S \subset \mathbb{H}^{n}$ calculated in the gauge metric. Also we write $\mathcal{H}^{\alpha}$, resp. $\mathcal{H}_{\delta}^{\alpha}, 0<\delta \leq \infty$, for the $\alpha$-dimensional Hausdorff measure, resp. premeasure at scale $\delta$, in the gauge metric, and we write $\mathcal{H}_{E}^{\alpha}$ for the $\alpha$-dimensional (Euclidean) Hausdorff measure on $\mathbb{R}$. We note that $\mathcal{H}_{\infty}^{\alpha}$ coincides with the $\alpha$-dimensional Hausdorff content, defined as follows:

$$
\mathcal{H}_{\infty}^{\alpha}(A)=\inf \sum_{i=1}^{\infty} r_{i}^{\alpha},
$$

the infimum taken over all covers of $A$ with balls $\left\{B\left(p_{i}, r_{i}\right): i=1,2, \ldots\right\}$.

The CC metric $d_{c c}$ will not be used in any quantitative sense, apart from a brief occurrence in Example 6.5.

The Heisenberg group $\mathbb{H}^{n}$ is equipped with a natural conformal inversion

$$
j_{\mathbb{H}}(z, t)=\left(\frac{-z}{|z|^{2}-i t}, \frac{-t}{\|(z, t)\|_{\mathbb{H}}^{4}}\right) .
$$

Note that $\left\|j_{\mathbb{H}}(\cdot)\right\|_{\mathbb{H}}=\|\cdot\|_{\mathbb{H}}^{-1}$ and $j_{\mathbb{H}} \circ \delta_{r}=\delta_{1 / r} \circ j_{\mathbb{H}}$ for $r>0$.

2.3. Quasiconformal maps. Let $\Omega$ be a domain in $\mathbb{H}^{n}$. A homeomorphism $F$ : $\Omega \rightarrow F(\Omega) \subset \mathbb{H}^{n}$ is $K$-quasiconformal ( $K$ - $Q C$ for short) if

$$
H_{F}(p):=\limsup _{r \rightarrow 0} \frac{\sup \left\{d_{\mathbb{H}}(F(p), F(q)): d_{\mathbb{H}}(p, q)=r\right\}}{\inf \left\{d_{\mathbb{H}}(F(p), F(q)): d_{\mathbb{H}}(p, q)=r\right\}} \leq K
$$

for every $p \in \Omega$. If $F$ is $K$-QC for some $K<\infty$ we say that $F$ is quasiconformal or $Q C$. We use conformal as a synonym for 1-quasiconformal.

Each $C^{2}$ smooth quasiconformal map $F$ is a contact map which means that the pullback of the contact form $\omega$ under $F$ is a nonvanishing multiple of $\omega$ : $F^{*} \omega=\lambda \omega$. $F$ is called orientation-preserving if $\lambda>0$ and orientation-reversing if $\lambda<0$. According to Theorem 8 of [31, sufficiently smooth 2 orientation-preserving conformal maps on a domain in $\mathbb{H}^{n}$ are precisely the restrictions of compositions of left translations $p \mapsto p_{0} \cdot p, p_{0} \in \mathbb{H}^{n}$, dilations $p \mapsto \delta_{r}(p), r>0$, rotations $p \mapsto R_{A}(p)$, $A \in U(n)$, and the conformal inversion $3(p)=j_{\mathbb{H}}(p)$, defined on $\Omega=\mathbb{H}^{n} \backslash\{o\}$.

The theory of quasiconformal maps in $\mathbb{H}^{n}$ is due to Korányi and Reimann [31] and 32]; see also Heinonen [23, and Heinonen-Koskela 26] for extensions to metric measure spaces of bounded geometry. Quasiconformal maps in Carnot groups first arose in the context of Mostow's rigidity theorem [38. It is well known that the class

\footnotetext{
${ }^{2}$ After work of Capogna [15, 16] and Capogna-Cowling [17, it is now known that the smoothness criterion is automatically satisfied for 1-quasiconformal maps, even in arbitrary Carnot groups.

${ }^{3}$ The 1-quasiconformality of $F=j_{\mathbb{H}}$ is an easy exercise using the identity (2.5).
} 
of quasiconformal homeomorphisms of $\mathbb{H}^{n}$ coincides with the class of quasisymmetric homeomorphisms. A homeomorphism $F: X \rightarrow Y$ between metric spaces $(X, d)$ and $\left(Y, d^{\prime}\right)$ is called quasisymmetric if there is a homeomorphism $\eta:[0, \infty) \rightarrow[0, \infty)$ so that

$$
d\left(x_{1}, x_{2}\right) \leq t d\left(x_{1}, x_{3}\right) \quad \Rightarrow \quad d^{\prime}\left(F\left(x_{1}\right), F\left(x_{2}\right)\right) \leq \eta(t) d^{\prime}\left(F\left(x_{1}\right), F\left(x_{3}\right)\right)
$$

for all $x_{1}, x_{2}, x_{3} \in X$ and $t>0$.

Korányi and Reimann [31] and 32] identified the infinitesimal generators of flows of smooth quasiconformal homeomorphisms of $\mathbb{H}^{n}$. According to [32, §5], for each $u \in C^{\infty}\left(\mathbb{H}^{n}\right)$ the vector field

$$
V:=u \cdot T+\frac{1}{4} \sum_{j=1}^{n}\left(\left(X_{j} u\right) Y_{j}-\left(Y_{j} u\right) X_{j}\right)
$$

generates a flow $\left(F_{s}\right)_{s \in \mathbb{R}}$ of $C^{\infty}$ contact homeomorphisms of $\mathbb{H}^{n}$. The coefficient of quasiconformality $K_{s}$ for $F_{s}$ can be bounded above in terms of $n, s$ and the expressions $\left\|X_{k} X_{j} u-Y_{k} Y_{j} u\right\|_{\infty},\left\|X_{k} Y_{j} u+Y_{k} X_{j} u\right\|_{\infty}, j, k=1, \ldots, n$. (See Proposition 25 in [32.)

Example 2.5. Let $p_{0}=\left(z_{0}, t_{0}\right) \in \mathbb{H}^{n}$ and define $u_{p_{0}} \in C^{\infty}\left(\mathbb{H}^{n}\right)$ by $u_{p_{0}}(z, t)=$ $\left\langle z_{0}, z\right\rangle-\frac{1}{2}\left(t-t_{0}\right)$. By (2.4) the associated vector field is

$$
V_{p_{0}}=\frac{1}{4} \sum_{i=1}^{n}\left(\left(x_{0 i}-x_{i}\right) \frac{\partial}{\partial x_{i}}+\left(y_{0 i}-y_{i}\right) \frac{\partial}{\partial y_{i}}\right)+\frac{1}{2}\left(\left\langle z_{0}, z\right\rangle-t+t_{0}\right) \frac{\partial}{\partial t},
$$

where $z_{0}=\left(x_{01}+i y_{01}, \ldots, x_{0 n}+i y_{0 n}\right)$. The associated flow of mappings is

$$
F_{p_{0}, s}(p)=p_{0} \cdot \delta_{\exp (-s / 4)}\left(p_{0}^{-1} \cdot p\right)
$$

which is a flow of conformal homotheties with common fixed point at $p_{0}$. Note that $X_{k} X_{j} u_{p_{0}}, Y_{k} Y_{j} u_{p_{0}}$ and $X_{k} Y_{j} u_{p_{0}}+Y_{k} X_{j} u_{p_{0}}$ are identically equal to zero for all $j, k=1, \ldots, n$.

2.4. Assouad dimension redux. Similarities of $\mathbb{H}^{n}$ are bi-Lipschitz and thus preserve Assouad dimension. Luukkainen [35, Theorem A.10(1)] proved that Assouad dimension of subsets of $\mathbb{R}^{n}$ is invariant under inversion in the unit sphere $S^{n-1}$. We establish a Heisenberg analog.

Proposition 2.6. For any set $S \subset \mathbb{H}^{n} \backslash\{o\}, \operatorname{dim}_{A} S=\operatorname{dim}_{A} j_{\mathbb{H}}(S)$.

Proof. The key ingredient in the proof is the following analog of a classical Euclidean inversion relation:

$$
d_{\mathbb{H}}\left(j_{\mathbb{H}}(p), j_{\mathbb{H}}(q)\right)=\frac{d_{\mathbb{H}}(p, q)}{\|p\|_{\mathbb{H}}|| q \|_{\mathbb{H}}} .
$$

This is a straightforward computation; see [18, (2.15)]. With (2.5) in hand the proof of Proposition 2.6 follows the lines of [35, Theorem A.10(1)]. By Proposition 2.1 (i) we may work with the Korányi metric $d_{\mathbb{H}}$. Since $j_{\mathbb{H}}$ is an involution, it suffices to show that $S$ is $s$-homogeneous whenever $j_{\mathbb{H}}(S)$ is $s$-homogeneous. When $s=0$ this is trivial ( $S$ is 0 -homogeneous if and only if it is finite), so assume $s>0$. Let $\Lambda$ be a finite subset of $S$ with $\alpha \leq d_{\mathbb{H}}(p, q) \leq \beta$ for all distinct $p, q \in \Lambda$. Set $A=\min _{p \in \Lambda}\|p\|_{\mathbb{H}}$ and $B=\max _{p \in \Lambda}\|p\|_{\mathbb{H}}$. By (2.5),

$$
\frac{\alpha}{B^{2}} \leq d_{\mathbb{H}}\left(j_{\mathbb{H}}(p), j_{\mathbb{H}}(q)\right) \leq \frac{\beta}{A^{2}}
$$


for all distinct $p, q \in \Lambda$. Hence

$$
\# \Lambda=\# j_{\mathbb{H}}(\Lambda) \leq C_{s}(B / A)^{2 s}(\beta / \alpha)^{s},
$$

where $C_{s}$ denotes the $s$-homogeneity constant for $j_{\mathbb{H}}(S)$. If $\beta \leq A$ we have $B \leq$ $A+\beta \leq 2 A$ whence $\# \Lambda \leq 4^{s} C_{s}(\beta / \alpha)^{s}$. If $\beta>A$ we choose $k \in \mathbb{N}$ with $2^{k-1} A \leq$ $\beta<2^{k} A$ and decompose $\Lambda$ in a union of $k+1$ sets $\Lambda_{i}$ by intersecting with the annuli $B_{\mathbb{H}}\left(o, 2^{i} A\right) \backslash \overline{B_{\mathbb{H}}\left(o, 2^{i-1} A\right)}, i=1, \ldots, k+1$. Repeating the above argument for each $i$ and summing leads to the estimate $\# \Lambda \leq 64^{s} /\left(2^{s}-1\right) C_{s}(\beta / \alpha)^{s}$; see the proof of Theorem A.10(1) in [35] for further details. Combining the above cases, we see that $\Lambda$ is $s$-homogeneous.

In view of Proposition 2.6 and Korányi and Reimann's classification of the conformal maps in $\mathbb{H}^{n}$, we have the following:

Corollary 2.7. Assouad dimension is a conformal invariant in $\mathbb{H}^{n}$.

It is well known that quasiconformal maps can distort dimension. In the Heisenberg group and for the Assouad dimension we have the following:

Theorem 2.8. For any $n \geq 1$ and any $0<\alpha<\beta<2 n+2$, there are compact sets $S_{\alpha}, S_{\beta} \subset \mathbb{H}^{n}$ and a quasiconformal homeomorphism $F$ of $\mathbb{H}^{n}$ so that $\operatorname{dim}_{A} S_{\alpha}=\alpha$, $\operatorname{dim}_{A} S_{\beta}=\beta$, and $F\left(S_{\alpha}\right)=S_{\beta}$.

The analogous theorem for Hausdorff dimension is due to Balogh [4, following the original result of Gehring and Väisälä in Euclidean space 21. Theorem 2.8 is an immediate consequence of the $\mathbb{H}^{n}$ version of [4, Theorem 1.1] in view of the Ahlfors regularity of the relevant examples $S_{\alpha}, S_{\beta}$.

For $K$-dependent bounds on distortion of Assouad dimension by quasiconformal maps, see Theorem 4.6 .

\section{Fractal GeOMEtry IN $\mathbb{H}^{n}$}

3.1. The Heisenberg cube. Throughout this paper we make extensive use of selfsimilar tilings of $\mathbb{H}^{n}$. Strichartz [42] and [43, first considered dyadic fractal tilings in nilpotent stratified Lie groups; see also Gelbrich [22. Almost sure dimension formulas for horizontal self-similar and self-affine fractals in the first Heisenberg group were established in [6]; see [5] for further results. Fractal geometry in general Carnot groups, with application to Gromov's dimension comparison problem, has been studied in [7] and [8].

Our primary tool is a class of tilings of $\mathbb{H}^{n}$ which generalizes the construction in 42 and [43. We begin with a description of the basic tile.

Example 3.1. Fix an odd positive integer $b \geq 2 n+1$, and consider the iterated function system in $\mathbb{H}^{n}$ generated by the following collection of $b^{2 n+2}$ contractive similarities:

$$
F_{\mathbf{k}, l}: \mathbb{H}^{n} \rightarrow \mathbb{H}^{n}, \quad F_{\mathbf{k}, l}(p)=p_{\mathbf{k}, l} \cdot \delta_{1 / b}(p),
$$

where $p_{\mathbf{k}, l}=\left(z_{\mathbf{k}}, t_{l}\right)$ with

$$
z_{\mathbf{k}}=\left(\frac{k_{1}-\frac{b+1}{2}}{b}+i \frac{k_{n+1}-\frac{b+1}{2}}{b}, \ldots, \frac{k_{n}-\frac{b+1}{2}}{b}+i \frac{k_{2 n}-\frac{b+1}{2}}{b}\right),
$$


$\mathbf{k}=\left(k_{1}, \ldots, k_{2 n}\right) \in\{1, \ldots, b\}^{2 n}$, and

$$
t_{l}=\frac{l-\frac{b^{2}+1}{2}}{b^{2}}
$$

$l \in\left\{1, \ldots, b^{2}\right\}$. Each map $F_{\mathbf{k}, l}$ is a similarity map of $\mathbb{H}^{n}$ with contraction ratio $\frac{1}{b}<1$, hence the collection $\left\{F_{\mathbf{k}, l}\right\}$ defines a unique nonempty compact invariant set $T_{o} \subset \mathbb{H}^{n}$ characterized by the identity

$$
T_{o}=\bigcup_{\mathbf{k}, l} F_{\mathbf{k}, l}\left(T_{o}\right) .
$$

Writing $f_{\mathbf{k}}: \mathbb{C}^{n} \rightarrow \mathbb{C}^{n}$ for the first-layer projection of $F_{\mathbf{k}, l}: f_{\mathbf{k}}(z)=z_{\mathbf{k}}+\frac{1}{b} z$, $z \in \mathbb{C}^{n}$, we note that $\pi\left(T_{o}\right)=\left[-\frac{1}{2}, \frac{1}{2}\right]^{2 n}$ is the invariant set for the self-similar IFS $\left\{f_{\mathbf{k}}\right\}$ on $\mathbb{C}^{n}$.

Remark 3.2. We may identify $\mathbb{H}^{n}$ with $\mathbb{R}^{2 n+1}$ and view the IFS $\left\{F_{\mathbf{k}, l}\right\}$ as a self-affine IFS in $\mathbb{R}^{2 n+1}$ equipped with the Euclidean metric $d_{E}$. Thus $T_{o}$ is an Euclidean selfaffine set. According to Lemma 3.3 below, $o$ is an interior point of $T_{o}$. Thus $T_{o}$ has (Hausdorff or Assouad) dimension $2 n+2$ in $\left(\mathbb{H}^{n}, d_{c c}\right)$, and has dimension $2 n+1$ in $\left(\mathbb{R}^{2 n+1}, d_{E}\right)$. By Theorem 1.1 in 34, $T_{o}$ coincides with the closure of its interior, and $\partial T_{o} \subset \mathbb{R}^{2 n+1}$ is a null set for Lebesgue measure. Observe that

$$
\partial T_{o}=Q^{+} \cup Q^{-} \cup W
$$

where $Q^{ \pm}$are vertical translates of the Heisenberg square $Q$ which is defined as the invariant set for the IFS $\left\{F_{\mathbf{k},\left(b^{2}+1\right) / 2}\right\}_{\mathbf{k} \in\{1, \ldots, b\}^{2 n}}$. In fact, $Q^{+}$is the invariant set for the IFS $\left\{F_{\mathbf{k}, b^{2}}\right\}_{\mathbf{k}}$, while $Q^{-}$is the invariant set for the IFS $\left\{F_{\mathbf{k}, 1}\right\}_{\mathbf{k}}$. $Q$ is a sub-Riemannian self-similar fractal whose Hausdorff dimension (in either the CC or Euclidean metric) equals $2 n$; see [5] and [42] for further information. The set $W$ (the walls of $T_{o}$ ) is a union of portions of vertical hyperplanes; $W$ has CC Hausdorff dimension $2 n+1$ and Euclidean Hausdorff dimension $2 n$.

Lemma 3.3. $B\left(o, r_{\text {in }}\right) \subset T_{o} \subset \overline{B\left(o, r_{\text {out }}\right)}$ where

$$
r_{i n}:=\sqrt{\frac{1}{4 n}-\frac{1}{2 b+2}}
$$

and

$$
r_{\text {out }}:= \begin{cases}\sqrt{\frac{1}{4}+\frac{1}{2 b+2}}, & n=1, \\ \frac{1}{2}, & n \geq 2 .\end{cases}
$$

Note that $r_{\text {in }}>0$ and $r_{\text {out }} \leq \frac{3}{4}$ for all $n$, since $b \geq 2 n+1$.

In the proof, we use the following representation formula for points in $T_{o}$. Compare (2.8) in [42. We denote by $p:\{1, \ldots, b\}^{2 n} \times\left\{1, \ldots, b^{2}\right\} \rightarrow T_{o}$ the canonical symbolic representation map:

$$
\begin{gathered}
p\left(\left(\mathbf{k}^{(1)}, l^{(1)}\right),\left(\mathbf{k}^{(2)}, l^{(2)}\right), \ldots\right)=\lim _{m \rightarrow \infty} F_{\mathbf{k}^{(1)}, l^{(1)}} \circ \cdots \circ F_{\mathbf{k}^{(m)}, l^{(m)}}(o) \\
=p_{\mathbf{k}^{(1)}, l^{(1)}} \cdot \delta_{1 / b}\left(p_{\mathbf{k}^{(2)}, l^{(2)}}\right) \cdot \delta_{1 / b^{2}}\left(p_{\mathbf{k}^{(3)}, l^{(3)}}\right) \cdots .
\end{gathered}
$$

If

$$
z=\lim _{m \rightarrow \infty} f_{\mathbf{k}^{(1)}} \circ \cdots \circ f_{\mathbf{k}^{(m)}}(0)=z_{\mathbf{k}^{(1)}}+\frac{1}{b} z_{\mathbf{k}^{(2)}}+\frac{1}{b^{2}} z_{\mathbf{k}^{(3)}}+\cdots=\sum_{j=1}^{\infty} \frac{1}{b^{j-1}} z_{\mathbf{k}^{(j)}}
$$


is a point in $\pi\left(T_{o}\right)=\left[-\frac{1}{2}, \frac{1}{2}\right]^{2 n}$, then the $t$-coordinate of the point in (3.2) is

$$
\sum_{j=1}^{\infty} \frac{t_{l^{(j)}}}{b^{2 j-2}}+2 \sum_{1 \leq i<j<\infty} \frac{\left\langle z_{\mathbf{k}^{(i)}}, z_{\mathbf{k}^{(j)}}\right\rangle}{b^{i+j-2}} .
$$

Proof of Lemma 3.3. By (3.3) and Remark 3.2 points in $Q^{+}$have symbolic representations as in (3.2) with $l^{(j)}=b^{2}$ for all $j \geq 1$. Then $t_{l^{(j)}}=\frac{b^{2}-1}{2 b^{2}}$ for all $j \geq 1$ and the $t$-coordinate of such a point is equal to

$$
\frac{b^{2}-1}{2 b^{2}} \sum_{j=1}^{\infty} \frac{1}{b^{2 j-2}}+2 \sum_{1 \leq i<j<\infty} \frac{\left\langle z_{\mathbf{k}^{(i)}}, z_{\mathbf{k}^{(j)}}\right\rangle}{b^{i+j-2}}=\frac{1}{2}+2 \sum_{1 \leq i<j<\infty} \frac{\left\langle z_{\mathbf{k}^{(i)}}, z_{\mathbf{k}^{(j)}}\right\rangle}{b^{i+j-2}} .
$$

By (2.1) this value is at least

$$
\begin{aligned}
\frac{1}{2}-4 n \sum_{1 \leq i<j<\infty} \frac{\left|z_{\mathbf{k}^{(i)}}\right|_{\infty}\left|z_{\mathbf{k}^{(j)}}\right|_{\infty}}{b^{i+j-2}} & \geq \frac{1}{2}-n \frac{(b-1)^{2}}{b^{2}} \sum_{1 \leq i<j<\infty} \frac{1}{b^{i+j-2}} \\
& =\frac{1}{2}-\frac{n}{b+1}=2 n r_{i n}^{2}>0
\end{aligned}
$$

and at most

$$
\frac{1}{2}+4 n \sum_{1 \leq i<j<\infty} \frac{\left|z_{\mathbf{k}^{(i)}}\right|_{\infty}\left|z_{\mathbf{k}^{(j)}}\right|_{\infty}}{b^{i+j-2}} \leq \frac{1}{2}+\frac{n}{b+1} \leq 2 n r_{\text {out }}^{2}
$$

for all $n$. Here we used the estimate $\left|z_{\mathbf{k}}\right|_{\infty} \leq(b-1) /(2 b)$, valid for all $\mathbf{k} \in$ $\{1, \ldots, b\}^{2 n}$. In other words, if $(z, t) \in Q^{+}$, then $|z|_{\infty} \leq \frac{1}{2}$ and $t \in\left[2 n r_{\text {in }}^{2}, 2 n r_{\text {out }}^{2}\right]$. Similarly, if $(z, t) \in Q^{-}$, then $|z|_{\infty} \leq \frac{1}{2}$ and $t \in\left[-2 n r_{\text {out }}^{2},-2 n r_{i n}^{2}\right]$. Since $r_{\text {in }} \leq \frac{1}{2} \leq$ $r_{\text {out }}$ for all $n$ and $\partial T_{o}=Q^{+} \cup Q^{-} \cup W$, we conclude that $B\left(o, r_{\text {in }}\right) \subset T_{o} \subset \overline{B\left(o, r_{\text {out }}\right)}$ as desired.

3.2. Strichartz-type tilings in $\mathbb{H}^{n}$. Let $\mathbb{H}_{\mathbb{Z}}^{n}=\left\{(z, t) \in \mathbb{H}^{n}: z \in \mathbb{Z}^{n}+i \mathbb{Z}^{n}, t \in \mathbb{Z}\right\}$ denote the integral Heisenberg group. The defining equation (3.1) for $T_{o}$ can be rewritten as the tiling equation

$$
\delta_{b}\left(T_{o}\right)=\bigcup_{p \in D} p \cdot T_{o}
$$

where $p \cdot S:=\{p \cdot q: q \in S\}$ and $D$ denotes the set of points $p=(z, t)$ in $\mathbb{H}_{\mathbb{Z}}^{n}$ for which $|z|_{\infty} \leq \frac{b-1}{2}$ and $|t| \leq \frac{b^{2}-1}{2}$. Iterating and passing to the limit, we obtain

$$
\mathbb{H}^{n}=\bigcup_{p \in \mathbb{H}_{\mathbb{Z}}^{n}} p \cdot T_{o}
$$

thus $T_{o}$ generates a self-similar tiling of $\left(\mathbb{H}^{n}, d\right)$. If $p, q \in \mathbb{H}_{\mathbb{Z}}^{n}$ are distinct, then $p \cdot T_{o}$ and $q \cdot T_{o}$ have disjoint interiors 4 For $m \in \mathbb{Z}$ and $p \in \delta_{b^{m}} \mathbb{H}_{\mathbb{Z}}^{n}$, let

$$
T\left(p, b^{m}\right):=p \cdot \delta_{b^{m}}\left(T_{o}\right) ;
$$

then

$$
\mathbb{H}^{n}=\bigcup_{p \in \delta_{b} m \mathbb{H}_{\mathbb{Z}}^{n}} T\left(p, b^{m}\right)
$$

\footnotetext{
${ }^{4}$ Since $\pi\left((z, t) \cdot T_{o}\right) \subset z+\left[-\frac{1}{2}, \frac{1}{2}\right]^{2 n}$ and $\bigcup_{\iota \in \mathbb{Z}}(0, \iota) \cdot T_{o}=\left[-\frac{1}{2}, \frac{1}{2}\right]^{2 n} \times \mathbb{R}$.
} 
with disjoint interiors. Note that $T_{o}=T(o, 1)$. We write

$$
\mathcal{T}_{m}=\left\{T\left(p, b^{m}\right): p \in \delta_{b^{m}} \mathbb{H}_{\mathbb{Z}}^{n}\right\}
$$

for the set of tiles in (3.4), and $\mathcal{T}_{*}=\bigcup_{m \in \mathbb{Z}} \mathcal{T}_{m}$.

$\mathcal{T}_{m}$ refines $\mathcal{T}_{m+1}$ in the sense that each element of $\mathcal{T}_{m}$ is contained entirely within a unique element of $\mathcal{T}_{m+1}$. We call $T \in \mathcal{T}_{m}$ a child of $T^{\prime} \in \mathcal{T}_{m+1}$ (and $T^{\prime}$ the parent of $T$ ) if $T \subset T^{\prime}$. From Lemma 3.3 we deduce the inclusions

$$
B\left(p, r_{\text {in }} b^{m}\right) \subset T\left(p, b^{m}\right) \subset \overline{B\left(p, r_{\text {out }} b^{m}\right)}
$$

for all $m \in \mathbb{Z}$ and all $p \in \delta_{b^{m}} \mathbb{H}_{\mathbb{Z}}^{n}$.

We will use the following relation defined on $\mathcal{T}_{m}$. We say that $T\left((z, t), b^{m}\right)$ and $T\left(\left(z^{\prime}, t^{\prime}\right), b^{m}\right)$ are adjacent if $\left|z-z^{\prime}\right|_{\infty} \leq b^{m}$ and $\left|t-t^{\prime}\right| \leq 18 n b^{2 m}$. Each tile in $\mathcal{T}_{m}$ is adjacent to

$$
N_{0}=3^{2 n}(36 n+1)
$$

neighbors in $\mathcal{T}_{m}$. The significance of this notion is clarified in the following lemma.

Lemma 3.4. The distance between any two non-adjacent tiles in $\mathcal{T}_{m}$ is at least $2\left(1-r_{\text {out }}\right) b^{m} \geq \frac{1}{2} b^{m}$, and the distance between any two adjacent tiles in $\mathcal{T}_{m}$ is at most $2 r_{\text {out }}(36 n+1) b^{m} \leq 60 n b^{m}$.

Proof. Let $T=T\left(p, b^{m}\right)=T\left((z, t), b^{m}\right)$ and $T^{\prime}=T\left(p^{\prime}, b^{m}\right)=T\left(\left(z^{\prime}, t^{\prime}\right), b^{m}\right)$ be two non-adjacent tiles in $\mathcal{T}_{m}$. If $\left|z-z^{\prime}\right|_{\infty} \geq 2 b^{m}$, then

$$
\begin{aligned}
\operatorname{dist}\left(T, T^{\prime}\right) & \geq d\left(p, p^{\prime}\right)-2 r_{\text {out }} b^{m} \\
& \geq\left|z-z^{\prime}\right|_{\infty}-2 r_{\text {out }} b^{m} \geq 2\left(1-r_{\text {out }}\right) b^{m},
\end{aligned}
$$

while if $\left|z-z^{\prime}\right|_{\infty} \leq b^{m}$ and $\left|t-t^{\prime}\right|>18 n b^{2 m}$, then also

$$
\begin{aligned}
\operatorname{dist}\left(T, T^{\prime}\right) & \geq d\left(p, p^{\prime}\right)-2 r_{\text {out }} b^{m} \\
& \geq d\left(p,\left(z, t^{\prime}\right)\right)-d\left(\left(z, t^{\prime}\right), p^{\prime}\right)-2 r_{\text {out }} b^{m} \\
& \geq \frac{\left|t-t^{\prime}\right|^{1 / 2}}{\sqrt{2 n}}-\left|z-z^{\prime}\right|_{\infty}-2 r_{\text {out }} b^{m} \geq 2\left(1-r_{\text {out }}\right) b^{m} .
\end{aligned}
$$

On the other hand, if $T$ and $T^{\prime}$ are adjacent, then there exists a chain of tiles $T=T_{0}, T_{1}, \ldots, T_{N}=T^{\prime}$ in $\mathcal{T}_{m}$ with $N \leq 36 n+2$ and $T_{i} \cap T_{i-1} \neq \emptyset$ for all $i=1, \ldots, N$. Then

$$
\operatorname{dist}\left(T, T^{\prime}\right) \leq \sum_{i=1}^{N-1} \operatorname{diam} T_{i} \leq(N-1)\left(2 r_{\text {out }} b^{m}\right)
$$

as desired.

Let $T$ be an element of $\mathcal{T}_{m}$. We define the star of $T$ to be the set

$$
\operatorname{star}(T)=\bigcup\left\{T^{\prime} \in \mathcal{T}_{m}: T^{\prime} \text { is adjacent to } T\right\} .
$$

By Lemma 3.4.

$$
\operatorname{diam}(\operatorname{star}(T)) \leq 4 r_{\text {out }}(36 n+1) b^{m} \leq 120 n b^{m}
$$

for all $T \in \mathcal{T}_{m}$. 


\section{Porosity, sparseness, And Assouad dimension}

The notion of porosity is a common theme in geometric function theory and complex dynamics. A set $S$ in a metric space $(X, d)$ is called porous in $X$ if there exists a constant $c>0$ so that every ball $B(x, r)$ contains a smaller ball $B(z, c r)$ which is disjoint from $S$.

Roughly speaking, porous sets avoid subballs of comparable size within metric balls at every location and scale. For our purposes, it will be helpful to refine the notion of porosity to obtain greater control over the location of the omitted subball. The following definition is formulated using Strichartz tilings.

Definition 4.1. Let $b \geq 2 n+1$ be an odd integer and let $1 \leq k<b^{2 n+2}$. A set $S \subset \mathbb{H}^{n}$ is $(b, k)$-sparse if for $T \in \mathcal{T}_{*}$, the set $S$ has nonempty intersection with at most $k$ children of $T$.

See Definition 3.2 in [46] for the analogous notion in Euclidean space. A $(b, k)$ sparse set is evidently $\left(b^{j}, k^{j}\right)$-sparse for each $j \in \mathbb{N}$.

Remark 4.2. It is an easy exercise to show that $S$ is porous in $\mathbb{H}^{n}$ if and only if it is $\left(b, b^{2 n+2}-1\right)$-sparse for some sufficiently large $b$. Moreover, the porosity constant $c$ and the parameter $b$ can be chosen to depend only on each other and on $n$.

We now relate bounds for the Assouad dimension with the sparseness criterion. Compare Proposition 3.3 in [46].

Proposition 4.3. If $S \subset \mathbb{H}^{n}$ is $(b, k)$-sparse, then $S$ is $s$-homogeneous with $s=$ $\log k / \log b$. Conversely, if $\operatorname{dim}_{A} S<2 n+2$ and $s \in\left(\operatorname{dim}_{A} S, 2 n+2\right)$, then there exists $b \geq 2 n+1$ sufficiently large so that $S$ is $(b, k)$-sparse for some $k \leq b^{s} \leq$ $b^{2 n+2}-1$.

As a corollary we obtain the equivalence of porosity with a bound on the Assouad dimension. Compare Theorem 5.2 in 35.

Corollary 4.4. $S \subset \mathbb{H}^{n}$ is porous if and only if $\operatorname{dim}_{A} S<2 n+2$. The porosity constant $c$ depends only a choice of $s \in\left(\operatorname{dim}_{A} S, 2 n+2\right)$ and the s-homogeneity constant $C_{s}$. Conversely, $\operatorname{dim}_{A} S$ is bounded above by a constant depending only on $n$ and the porosity constant $c$.

Proof of Corollary 4.4. First, suppose that $\operatorname{dim}_{A} S<2 n+2$. Then $S$ is $s$-homogeneous for some $s<2 n+2$. By Proposition 4.3, $S$ is $(b, k)$-sparse for some sufficiently large integer $b$ and $k \leq b^{s}<b^{2 n+2}$. Then $S$ is $\left(b, b^{2 n+2}-1\right)$-sparse and hence porous by Remark 4.2 .

Conversely, assume that $S$ is porous and hence $\left(b, b^{2 n+2}-1\right)$-sparse. Then $S$ is $s$-homogeneous with

$$
s=\frac{\log \left(b^{2 n+2}-1\right)}{\log b}<2 n+2
$$

and hence $\operatorname{dim}_{A} S<2 n+2$.

Before proving Proposition 4.3, we state an application to dilatation-dependent bounds for quasisymmetric distortion of Assouad dimension. We require, in addition, the quasisymmetric invariance of porosity. The latter result holds in the general setting of Ahlfors regular Loewner metric measure spaces, as in the following theorem. Recall that a metric measure space $(X, d, \mu)$ is called Loewner (with exponent $Q)$ if there exists a decreasing function $\varphi:(0, \infty) \rightarrow(0, \infty)$ so that the 
$Q$-modulus of the family $\Gamma$ of curves joining two nondegenerate continua $E, F \subset X$ verifies the inequality

$$
\operatorname{Mod}_{Q} \Gamma \geq \varphi\left(\frac{\operatorname{dist}(E, F)}{\min \{\operatorname{diam} E, \operatorname{diam} F\}}\right)
$$

for every pair $E, F$; see [26] or 25] for more information and the definition of the modulus of a curve family. The Heisenberg group $\mathbb{H}^{n}$ (or any Carnot group equipped with Carnot-Carathéodory metric and Haar measure) is an Ahlfors regular Loewner space; see [26, section 6.2], 23] or [24].

Theorem 4.5. Let $(X, d, \mu)$ and $\left(Y, d^{\prime}, \mu^{\prime}\right)$ be locally compact Ahlfors $Q$-regular Loewner metric measure spaces, $Q>1$, and let $F: X \rightarrow Y$ be $\eta$-quasisymmetric. If $S \subset X$ is c-porous in $X$ for some c $>0$, then $F(S)$ is c'-porous in $Y$ for some $c^{\prime}$ depending only on $c, \eta, Q$, and the regularity data of $X$ and $Y$.

Theorem 4.5 is a straightforward application of the Loewner condition and modulus estimates. It is rather more difficult to prove the invariance of porosity under quasisymmetric embeddings $F: S \hookrightarrow Y$ defined only on $S$; see 48 for the Euclidean case. We do not need such stronger results in our context.

Proof. We assume that $X$ and $Y$ are unbounded which suffices for our application to the Heisenberg group. The bounded case is similar. The inverse map $F^{-1}: Y \rightarrow X$ is $\eta^{\prime}$-quasisymmetric for a suitable $\eta^{\prime}$ depending only on $\eta$. Suppose that $S$ is $c$ porous in $X$ but $F(S)$ is not $c^{\prime}$-porous in $Y$. We will show that $c^{\prime}$ admits a uniform lower bound depending only on the data specified in the statement of the theorem. We may assume that $c^{\prime} \leq(4 \eta(1))^{-1} \leq \frac{1}{4}$. There exists a ball $B\left(y, r^{\prime}\right)$ in $Y$ so that $F(S) \cap B\left(y, r^{\prime}\right)$ is $c^{\prime} r^{\prime}$-dense in $B\left(y, r^{\prime}\right)$. Choose $r>0$ so that

$$
B(x, r) \subset F^{-1} B\left(y, r^{\prime} / 2\right) \subset F^{-1} B\left(y, r^{\prime}\right) \subset B\left(x, H^{\prime} r\right),
$$

where $x=F^{-1}(y)$ and $H^{\prime}=\eta^{\prime}(2)$. By porosity of $S$, there exists a subball $B(z, c r) \subset B\left(x, \frac{1}{2} r\right)$ which is disjoint from $S$. Choose $t>0$ so that

$$
B(w, t) \subset F B(z, c r) \subset B(w, H t),
$$

where $w=F(z) \in B\left(y, r^{\prime} / 2\right)$ and $H=\eta(1)$. Since $B(w, t)$ is disjoint from $F(S)$ and $B\left(w, c^{\prime} r^{\prime}\right)$ meets $F(S)$, we must have $t<c^{\prime} r^{\prime}$, so

$$
B(w, t) \subset F B(z, c r) \subset B\left(w, H c^{\prime} r^{\prime}\right) .
$$

Let $\Gamma$ denote the family of curves joining $B(z, c r)$ to $X \backslash B\left(x, H^{\prime} r\right)$. By the Loewner property of $(X, d, \mu)$ and the comparability of diam $B(x, r)$ and $r$,

$$
\operatorname{Mod}_{Q} \Gamma \geq \varphi\left(\frac{2 H^{\prime} r / c_{1}}{c_{1} c r}\right)=\varphi\left(\frac{2 H^{\prime}}{c c_{1}^{2}}\right)>0
$$

for some $c_{1}>0$ depending only on the regularity data of $X$. On the other hand, the curve family $F(\Gamma)$ is minorized by the curve family $\Gamma^{\prime}$ joining $B\left(w, H c^{\prime} r^{\prime}\right)$ to $Y \backslash B\left(w, r^{\prime} / 2\right)$. Then

$$
\operatorname{Mod}_{Q} F(\Gamma) \leq \operatorname{Mod}_{Q} \Gamma^{\prime} \leq C\left(\log \frac{1}{2 H c^{\prime}}\right)^{1-Q}
$$

for some constant $C$ depending only on the regularity data for $Y$, by a standard estimate for the $Q$-modulus (see [26, Lemma 3.14]). 
Every $\eta$-quasisymmetric homeomorphism between locally compact $Q$-regular metric measure spaces, $Q>1$, satisfies the geometric quasiconformality condition

$$
K^{-1} \operatorname{Mod}_{Q} \Gamma \leq \operatorname{Mod}_{Q} F(\Gamma) \leq K \operatorname{Mod}_{Q} \Gamma
$$

for all curve families $\Gamma$ and some constant $K \geq 1$ depending only on $\eta, Q$, and the regularity data of $X$ and $Y$; see [44. Thus

$$
0<\varphi\left(\frac{2 H^{\prime}}{c c_{1}^{2}}\right) \leq K C\left(\log \left(\frac{1}{2 H c^{\prime}}\right)\right)^{1-Q}
$$

so

$$
c^{\prime} \geq \frac{1}{2 H} \exp \left(-\left(\frac{1}{K C} \varphi\left(\frac{2 H^{\prime}}{c c_{1}^{2}}\right)\right)^{1 /(1-Q)}\right) .
$$

This completes the proof.

Combining Corollary 4.4 and Theorem 4.5 gives Theorem 1.2 and Corollary 1.3 from the introduction, which we restate in the following form:

Theorem 4.6. Let $F: \mathbb{H}^{n} \rightarrow \mathbb{H}^{n}$ be a K-quasiconformal homeomorphism, and let $S \subset \mathbb{H}^{n}$ satisfy $\operatorname{dim}_{A} S \in(0,2 n+2)$. Then

$$
0<\alpha \leq \operatorname{dim}_{A} F(S) \leq \beta<2 n+2
$$

where the constants $\alpha, \beta$ depend only on $K, n$, a constant $s \in\left(\operatorname{dim}_{A} S, 2 n+2\right)$ and the s-homogeneity constant $C_{s}$ for $S$.

In fact, $\alpha$ can be chosen to depend only on $K$ and $\operatorname{dim}_{A} S$; this follows from Proposition 5.1 in 46 and the fact that $F$ is power quasisymmetric.

The analog of Theorem 4.6 for Hausdorff dimension in Euclidean space is the well-known result of Gehring and Väisälä [21, Theorem 12]. In the case of Hausdorff dimension in the Heisenberg group, the relevant result is due to Balogh [4, Theorem 4.1]. For Assouad dimension in Euclidean space, see [46, Proposition 5.4].

We conclude this section with the proof of Proposition 4.3

Proof of Proposition 4.3. Suppose that $S$ is $(b, k)$-sparse with $k<b^{2 n+2}$; we will show that $S$ is $s$-homogeneous for $s=\log k / \log b<2 n+2$. Choose $0<\alpha \leq \beta<\infty$ and let $\Lambda$ be a finite subset of $S$ satisfying $\alpha \leq d(p, q) \leq \beta$ for all $p, q \in \Lambda, p \neq q$. We estimate the cardinality of $\Lambda$. Choose integers $m$ and $M$ so that $2 b^{m} \leq \alpha<2 b^{m+1}$ and $\frac{1}{2} b^{M-1} \leq \beta<\frac{1}{2} b^{M}$. We claim that

(i) $\Lambda$ is contained in at most $N_{0}$ elements of $\mathcal{T}_{M}$, and

(ii) any two distinct elements of $\Lambda$ lie in distinct elements of $\mathcal{T}_{m}$.

To prove (i), choose $p \in \Lambda$ and a tile $T \in \mathcal{T}_{M}$ containing $p$ and observe that $\Lambda \subset \operatorname{star}(T)$. Indeed, if $q \in \Lambda \cap T^{\prime}$ with $T$ and $T^{\prime}$ nonadjacent in $\mathcal{T}_{M}$, then

$$
\frac{1}{2} b^{M} \leq \operatorname{dist}\left(T, T^{\prime}\right) \leq d(p, q) \leq \beta<\frac{1}{2} b^{M} .
$$

Similarly, to prove (ii) we note that no two points of $\Lambda$ can lie in the same element of $\mathcal{T}_{m}$. Indeed, if $p, q \in \Lambda \cap T$ with $T \in \mathcal{T}_{m}$, then

$$
2 b^{m} \leq \alpha \leq d(p, q) \leq \operatorname{diam} T \leq 2 r_{\text {out }} b^{m}<2 b^{m}
$$

by (3.5). 
By (i), (ii) and the $(b, k)$-sparsity of $S$, we have $\# \Lambda \leq N_{0} k^{M-m}$. By the choice of $m$ and $M$,

$$
M-m \leq 2+\frac{\log (4 \beta / \alpha)}{\log b}
$$

Thus

$$
\# \Lambda \leq N_{0} k^{2+\log (4 \beta / \alpha) / \log b}=N_{0} k^{2} 4^{s}(\beta / \alpha)^{s} \leq(2 b)^{4 n+4} N_{0}(\beta / \alpha)^{s}
$$

which shows that $S$ is $s$-homogeneous.

Next, assume that $\operatorname{dim}_{A} S<2 n+2$ and $s \in\left(\operatorname{dim}_{A} S, 2 n+2\right)$. Choose $t \in$ $\left(\operatorname{dim}_{A} S, s\right)$ and let $C_{t}$ be the $t$-homogeneity constant of $S$. Let $b \geq 2 n+1$ be a large odd integer whose exact value will be determined momentarily. We consider the tilings $\mathcal{T}_{m}, m \in \mathbb{Z}$. For each $T \in \mathcal{T}_{m}$ we may divide the children of $T$ into at most $N_{0}$ families, each containing at least

$$
b^{2 n+2} / N_{0}
$$

elements, so that any two tiles in the same family are nonadjacent. Suppose that a collection of tiles $T_{1}, \ldots, T_{L}$ from one of these families each intersect $S$. Choose $p_{i} \in T_{i} \cap S$, and let $\Lambda=\left\{p_{1}, \ldots, p_{L}\right\}$. Since $S$ is $t$-homogeneous,

$$
L \leq C_{t}\left(\frac{\operatorname{diam} \Lambda}{\min \{d(p, q): p, q \in \Lambda, p \neq q\}}\right)^{t} \leq C_{t}\left(\frac{2 r_{\text {out }} b^{m}}{\frac{1}{2} b^{m-1}}\right)^{t} \leq C_{t}(3 b)^{t} .
$$

Summing over all families, we find that the total number of children of $T$ which can intersect $S$ is at most

$$
N_{0} C_{t}(3 b)^{t} \leq 9^{2 n+1}(36 n+1) C_{t} b^{t} .
$$

We now choose $b$ so large that

$$
9^{2 n+1}(36 n+1) C_{t} b^{t} \leq b^{s} \leq b^{2 n+2}-1
$$

then $S$ is $(b, k)$-sparse with $k \leq b^{s}$ as desired.

\section{Global conformal Assouad dimension in $\mathbb{H}^{n}$}

This section is devoted to the proof of Theorem 1.1, which we restate in expanded form in the following two propositions.

Proposition 5.1. For each $\alpha \in\{0\} \cup[1,2 n+2]$ there exists a bounded set $S \subset \mathbb{H}^{n}$ with

$$
G C \operatorname{dim}_{A} S=\operatorname{dim}_{A} S=G C \operatorname{dim}_{H} S=\operatorname{dim}_{H} S=\alpha .
$$

Proposition 5.2. If $S \subset \mathbb{H}^{n}$ satisfies $\operatorname{dim}_{A} S<1$, then $G C \operatorname{dim}_{A} S=0$.

Proposition 5.1 is a strengthened version of Theorem 1.3 in [4], which concerns Hausdorff dimension. When $1 \leq \alpha \leq 2 n+1$ the relevant examples from [4] (extended to $\mathbb{H}^{n}$ ) continue to function for Proposition 5.1. When $2 n+1<\alpha<2 n+2$, technical complications force us to use different examples. We use recent results from [1] and [2] on the regularity of the $\mathrm{CC}$ distance function to construct the desired examples; see Example 6.5 for details.

We prove Propositions 5.1 and 5.2 in subsections 5.1 and 5.2, respectively. 
5.1. Sets in $\mathbb{H}^{n}$ with prescribed global conformal Assouad dimension. In this subsection, we construct examples of sets $S \subset \mathbb{H}^{n}$ satisfying (5.1) for each $\alpha \in\{0\} \cup[1,2 n+2]$. The cases $\alpha=0, \alpha=1, \alpha=2 n+1$ and $\alpha=2 n+2$ are easily dealt with, considering finite sets, smooth bounded horizontal curves, boundaries of smooth domains (see Example 6.2) and closed balls. We treat the cases $1<\alpha<2 n+1$ and $2 n+1<\alpha<2 n+2$ in turn.

Case I $(\alpha \in(1,2 n+1))$. In this case, examples from 4 continue to serve; we briefly recall the construction and refer to 4 for the technical details.

We construct an $\alpha$-regular set $S \subset \mathbb{H}^{n}$ which verifies the conditions in Lemma 2.3. Let $S_{0}$ be a self-similar Ahlfors $(\alpha-1)$-regular Cantor set contained in the codimension two subspace $P=\left\{(z, t) \in \mathbb{H}^{n}: z_{1}=0\right\}$. Such a set can be constructed as the invariant set for an iterated function system satisfying the strong open set condition with fixed points in $P$; see $[8$ or Example 6.1 for more details. The Hausdorff measure $\mathcal{H}^{\alpha-1}$ restricted to $S_{0}$ (and suitably normalized) is an $(\alpha-1)$ regular measure. Set

$$
S=\left\{\left(t e_{1}, 0\right) \cdot p: p \in S_{0}, 0 \leq t \leq 1\right\},
$$

where $e_{1}=(1,0, \ldots, 0) \in \mathbb{C}^{n}$. We write $t * p$ for the point $\left(t e_{1}, 0\right) \cdot p$. We define a measure $\nu$ on $S$ by setting

$$
\nu(A * B)=\mathcal{L}^{1}(A) \mathcal{H}^{\alpha-1}(B)
$$

for $A \subset[0,1]$ and $B \subset S_{0}\left(\mathcal{L}^{1}\right.$ denotes Lebesgue measure on $\left.[0,1]\right)$ and extending to a measure on the $\sigma$-algebra generated by product sets of the type $A * B$. Then $\nu$ is an $\alpha$-regular measure on $S$; see the discussion on pp. 305-306 of 4] for the proof in the $\mathbb{H}^{1}$ case. The measure $\mathcal{H}^{\alpha-1}$ on $S_{0}$ lifts to a probability measure on the curve family

$$
\Gamma=\left\{\gamma_{p}\right\}_{p \in S_{0}}, \quad \gamma_{p}:[0,1] \rightarrow \mathbb{H}^{n}, \quad \gamma_{p}(t)=\left(t e_{1}, 0\right) \cdot p .
$$

The hypotheses of Lemma 2.3 are easily verified. This completes the proof in Case I.

Case II $(\alpha \in(2 n+1,2 n+2))$. As mentioned above, it is unclear whether the examples from [4] continue to serve in this case. Recall that Lemmas 5.1 and 5.2 of 4] show that certain Cantor sets of Heisenberg spheres are minimal for $G C \operatorname{dim}_{H}^{\mathbb{H}^{n}}$. In attempting to use the same example for $G C \operatorname{dim}_{A}^{\mathbb{H}^{n}}$ we encounter difficulties in showing that such sets have the prescribed Assouad dimension. Indeed, although these sets are constructed as a kind of product of Ahlfors regular "radial" and "spherical" sets, it is not clear whether they are in fact Ahlfors regular. Complications arise in estimating the measure near characteristic points of the spheres. We avoid this issue by replacing "Cantor sets of Heisenberg spheres" with "Cantor sets of smooth closed noncharacteristic hypersurfaces". We establish the Ahlfors regularity of such sets in Example 6.5. In what follows, we show that they are minimal for global conformal dimension.

Lemma 5.3. Let $S$ be a $C^{3}$ noncharacteristic hypersurface bounding a domain $\Omega$ in $\mathbb{H}^{n}$, let $\epsilon>0$ be as in Proposition 6.3 , let $D \subset[0, \epsilon)$ be an a-regular Cantor set $(0<a<1)$, and let $X=\bigcup_{r \in D} P^{+}(S, r)$ be the $(2 n+1+a)$-regular set constructed in Example 6.5. Then $G C \operatorname{dim}_{H}^{\mathbb{H}^{n}} X \geq 2 n+1+a$.

Combining Lemma 5.3 and Example 6.5 we deduce that $X$ is minimal for global conformal dimension (Hausdorff or Assouad), of dimension $2 n+1+a$. 
Proof of Lemma 5.3. Again, we adapt an argument from 44. Let $F$ be a quasiconformal homeomorphism of $\mathbb{H}^{n}$. Our first goal is to show that

$$
\inf _{r \in D} \mathcal{H}_{\infty}^{2 n+1}\left(F\left(P^{+}(S, r)\right)\right)>0,
$$

where $\mathcal{H}_{\infty}^{\alpha}$ denotes the $\alpha$-dimensional Hausdorff content; see (2.2).

Adapting an argument of Pansu (see p. 309 of [4]) we derive (5.2) from Pansu's isoperimetric inequality

$$
\mathcal{H}^{2 n+1}(\partial \Omega) \geq c|\Omega|^{\frac{2 n+1}{2 n+2}}
$$

valid for all bounded domains $\Omega \subset \mathbb{H}^{n}$ with piecewise $C^{2}$ boundary, for some absolute constant $c>0$; see [39] or [18] for a proof of (5.3) in the first Heisenberg group. Since $F\left(P^{+}(S, r)\right)$ is compact it suffices to consider finite covers $\left\{B_{1}, \ldots, B_{N}\right\}$ in (5.2). Writing $\Omega^{\prime}=B(S, r) \cup B_{1} \cup \cdots \cup B_{N} \supset \Omega$ and noting that $\partial \Omega^{\prime} \subset \bigcup_{i=1}^{N} \partial B_{i}$, we estimate

$$
\begin{aligned}
0 & <|F(\Omega)|^{(2 n+1) /(2 n+2)} \leq\left|F\left(\Omega^{\prime}\right)\right|^{(2 n+1) /(2 n+2)} \leq C \mathcal{H}^{2 n+1}\left(\partial \Omega^{\prime}\right) \\
& \leq C \sum_{i=1}^{N} \mathcal{H}^{2 n+1}\left(\partial B_{i}\right) \leq C \sum_{i=1}^{N} r_{i}^{2 n+1},
\end{aligned}
$$

which yields (5.2). The final inequality here comes from the Ahlfors $(2 n+1)$ regularity of $\partial B_{i}$; see Example 6.2.

Let $0<\eta<a / 2$ and let $\left\{B_{i}^{\prime}=B\left(p_{i}^{\prime}, r_{i}^{\prime}\right): i=1,2, \ldots\right\}$ be an arbitrary cover of $F(X)$. We may assume that the dilates $B\left(p_{i}^{\prime}, \frac{1}{5} r_{i}^{\prime}\right)$ are pairwise disjoint. Setting $p_{i}=F^{-1}\left(p_{i}^{\prime}\right)$, we deduce from the quasisymmetry of $F$ and the equivalence of $d$ and $d_{c c}$ the existence of a constant $H=H(F) \geq 1$ and radii $r_{i}>0$ so that

$$
B_{c c}\left(p_{i}, r_{i}\right) \subset F^{-1}\left(B\left(p_{i}^{\prime}, \frac{1}{5} r_{i}^{\prime}\right)\right) \subset F^{-1}\left(B\left(p_{i}^{\prime}, r_{i}^{\prime}\right)\right) \subset B_{c c}\left(p_{i}, H r_{i}\right)
$$

for all $i$. The balls $\left\{B_{c c}\left(p_{i}, H r_{i}\right): i=1,2, \ldots\right\}$ cover $X$, while the dilated balls $\left\{B_{c c}\left(p_{i}, \frac{1}{2} r_{i}\right): i=1,2, \ldots\right\}$ have disjoint closures. By (5.2),

$$
0<c \leq \mathcal{H}_{\infty}^{2 n+1}\left(F\left(P^{+}(S, r)\right)\right) \leq \sum_{i: P^{+}(S, r) \cap F^{-1}\left(B_{i}^{\prime}\right) \neq \emptyset}\left(r_{i}^{\prime}\right)^{2 n+1}
$$

for each $r \in D$. Integrating with respect to the $a$-regular measure $\mathcal{H}_{E}^{a}$ on $D$ yields

$$
\begin{aligned}
0<c & \leq \sum_{i}\left(r_{i}^{\prime}\right)^{2 n+1} \mathcal{H}_{E}^{a}\left(\left\{r \in D: P^{+}(S, r) \cap F^{-1}\left(B_{i}^{\prime}\right) \neq \emptyset\right\}\right) \\
& \leq \sum_{i}\left(r_{i}^{\prime}\right)^{2 n+1} \mathcal{H}_{E}^{a}\left(\left\{r \in D: P^{+}(S, r) \cap B_{c c}\left(p_{i}, H r_{i}\right) \neq \emptyset\right\}\right) \\
& =\sum_{i}\left(r_{i}^{\prime}\right)^{2 n+1} \mathcal{H}_{E}^{a}\left(\left\{r \in D:\left|r-\operatorname{dist}_{c c}\left(p_{i}, S\right)\right| \leq H r_{i}\right\}\right) \\
& \leq C \sum_{i}\left(r_{i}^{\prime}\right)^{2 n+1} r_{i}^{a} .
\end{aligned}
$$

Set $b=1+(2 n+1) /(a-\eta)>1+(2 n+1) / a$. Since $b a>2 n+1+a=\operatorname{dim}_{A} X$ we may choose $\delta>0$ sufficiently small so that $r_{i} \leq \delta$ implies $\sum_{i} r_{i}^{b a} \leq 1$; see [36. section 5.9] for details. By local uniform continuity of $F^{-1}$, we may choose 
$\epsilon>0$ sufficiently small so that $r_{i} \leq \delta$ whenever $r_{i}^{\prime} \leq \epsilon$. An application of Hölder's inequality gives

$$
0<c \leq\left(\sum_{i}\left(r_{i}^{\prime}\right)^{\frac{(2 n+1) b}{b-1}}\right)^{1-1 / b}\left(\sum_{i} r_{i}^{b a}\right)^{1 / b} \leq\left(\sum_{i}\left(r_{i}^{\prime}\right)^{2 n+1+a-\eta}\right)^{1-1 / b}
$$

from which we conclude

$$
\mathcal{H}^{2 n+1+a-\eta}(F(X)) \geq \mathcal{H}_{\epsilon}^{2 n+1+a-\eta}(F(X)) \geq c^{b /(b-1)}>0 .
$$

Thus $\operatorname{dim}_{H} F(X) \geq 2 n+1+a-\eta$. Taking the infimum over all quasiconformal homeomorphisms $F$ of $\mathbb{H}^{n}$ and letting $\eta \searrow 0$ completes the proof.

5.2. Global conformal Assouad dimension in $\mathbb{H}^{n}$ takes on no value between zero and one. Suppose that $S \subset \mathbb{H}^{n}$ satisfies $\operatorname{dim}_{A} S<1$. We aim to show that $G C \operatorname{dim}_{A} S=0$. According to Proposition 4.3, $S$ is $(b, k)$-porous for some large integer $b$ and $k \leq b^{s}$, where $\operatorname{dim}_{A} S<s<1$. By choosing $b \geq b_{0}$ for some sufficiently large $b_{0}=b_{0}(s)$, we may arrange that $k / b$ is as small as we please.

The crux of the proof of Proposition 5.2 resides in the following technical lemma.

Lemma 5.4. For each $s<1$ there exists $b_{0}=b_{0}(s)$ so that the following holds for all $b \geq b_{0}$ : Let $S$ be a subset of the standard b-adic Strichartz tile $T_{o}$, and assume that $S$ is $(b, k)$-sparse for some $k \leq b^{s}$. For each $m \leq 0$, there exists an integer $N_{m}$ and for each $j=1, \ldots, N_{m}$ there exist three collections $\mathcal{A}(m, j), \mathcal{B}(m, j), \mathcal{C}(m, j)$, each consisting of finitely many tiles in $\mathcal{T}_{m}$, satisfying the following conditions:

(i) $S$ is contained in $\bigcup_{j} \cup \mathcal{A}(m, j)$,

(ii) for each $m$ and $j$,

$$
\cup\{\operatorname{star}(T): T \in \mathcal{A}(m, j)\}=\cup \mathcal{B}(m, j)
$$

and

(iii) for each $m \leq-1$ and each $j$, there exists $j^{\prime}$ so that $\cup \mathcal{C}(m, j) \subset \mathcal{B}\left(m+1, j^{\prime}\right)$,

(iv) for each $m$ and $i \neq j, \cup \mathcal{C}(m, i)$ and $\cup \mathcal{C}(m, j)$ are disjoint,

(v) for each $m$ and $j$, there exists $T \in \mathcal{T}_{m+1}$ so that $\cup \mathcal{B}(m, j) \subset \operatorname{star}(T)$,

(vi) for each $m$ and each $T \in \mathcal{T}_{m}$ with $T \subset T_{o}, S$ has nonvoid intersection with at most $k$ of the sets $\cup \mathcal{A}(m, j), j=1, \ldots, N_{m}$.

Here for a collection $\mathcal{C}$ of sets, we write $\cup \mathcal{C}=\{p: p \in C$ for some $C \in \mathcal{C}\}$.

The separating annuli $\cup \mathcal{C}(m, j) \backslash \cup \mathcal{B}(m, j)$ which appear in the preceding proof will enable us to shrink the Assouad dimension of $S$ by implementing a KorányiReimann flow which conformally shrinks each of the sets $\cup \mathcal{B}(m, j)$ while leaving the complement of $\bigcup_{j} \cup \mathcal{C}(m, j)$ invariant. For technical reasons we work also with the associated annulus $\cup \mathcal{B}(m, j) \backslash \cup \mathcal{A}(m, j)$; the reason for this added complication in the proof stems from the appearance of $\operatorname{star}(T)$ in part (v) of the proof.

Before beginning the proof, we make some brief remarks. First, by (5.5) and Lemma 5.4(iv), no element of $\mathcal{B}(m, i)$ is adjacent to any element of $\mathcal{B}(m, j)$ when $i \neq j$. By Lemma $3.4^{5}$

$$
\operatorname{dist}(\cup \mathcal{B}(m, i), \cup \mathcal{B}(m, j)) \geq \frac{1}{2} b^{m} \quad \forall i \neq j .
$$

\footnotetext{
${ }^{5}$ We remind the reader that we are using the gauge metric $d$.
} 
Similarly, by Lemma 5.4(v) and (3.6),

$$
\operatorname{diam}(\cup \mathcal{B}(m, i)) \leq 120 n b^{m+1} \quad \forall i .
$$

Proof. Choose $b_{0}=b_{0}(s) \geq 1000 n$ and $b \geq b_{0}$ so that $k \leq b /(1000 n)$. We prove the result by induction on $m \leq 0$.

The case $m=0$ is trivial: choose $N_{0}=1$ and

$$
\mathcal{A}(0,1)=\left\{T_{o}\right\}, \quad \mathcal{B}(0,1)=\left\{\operatorname{star}\left(T_{o}\right)\right\}, \quad \mathcal{C}(0,1)=\left\{\operatorname{star}\left(\operatorname{star}\left(T_{o}\right)\right)\right\} .
$$

Suppose that the conclusion holds for some $m+1 \leq 0$; we show it for $m$. Fix a tile $T$ in $\mathcal{A}(m+1, j)$ for some $j \leq N_{m+1}$, and let $T_{1}, \ldots, T_{q}, q \leq k$, be the children of $T$ which meet $S$. Fix $i, 1 \leq i \leq q$, and define a sequence of annular shells $\mathcal{F}_{j}=\mathcal{F}_{j}\left(T, T_{i}\right), j \in\{0,1,2, \ldots\}$, as follows: $\mathcal{F}_{0}=\left\{T_{i}\right\}$ and for each $j \in \mathbb{N}, \mathcal{F}_{j}$ consists of all elements of $\mathcal{T}_{m} \backslash\left\{T_{i}\right\}$ which are adjacent to but not contained in an element of $\mathcal{F}_{0} \cup \cdots \cup \mathcal{F}_{j-1}$. Since $k \leq b /(1000 n)$ there exists $j(i), 1 \leq j(i) \leq b /(250 n)$, so that $\mathcal{F}_{j(i)}, \mathcal{F}_{j(i)+1}$ and $\mathcal{F}_{j(i)+2}$ consist entirely of tiles disjoint from $S$. We claim that

$$
\left(\cup \mathcal{F}_{0}\right) \cup \cdots \cup\left(\cup \mathcal{F}_{j(i)}\right) \subset \operatorname{star}(T)
$$

for each $i$. To see this, suppose that there exists a tile $T^{\prime}$ in $\mathcal{F}_{0} \cup \cdots \cup \mathcal{F}_{j(i)}$ whose parent is not adjacent to $T$. Then

$$
\frac{1}{2} b^{m+1} \leq \operatorname{dist}\left(T, \text { parent of } T^{\prime}\right) \leq \operatorname{dist}\left(T_{i}, T^{\prime}\right) .
$$

This distance is bounded above by the sum of $j(i)$ terms, each of which is the diameter of the star of an element of $\mathcal{T}_{m}$. By (3.6),

$$
\frac{1}{2} b^{m+1} \leq j(i)\left(120 n b^{m}\right) \leq \frac{12}{25} b^{m+1},
$$

which is a contradiction. We conclude that all tiles in $\mathcal{F}_{0} \cup \cdots \cup \mathcal{F}_{j(i)}$ have a parent in $\operatorname{star}(T)$, which immediately implies (5.6).

We say that a pair of tiles $T \in \mathcal{T}_{m+1}, T_{i} \in \mathcal{T}_{m}$ are an admissible pair if

- $T$ is contained in $\mathcal{A}(m+1, j)$ for some $j \leq N_{m+1}$,

- $T_{i} \cap S \neq \emptyset$, and

- $T_{i}$ is a child of $T$.

Repeat the construction in the preceding paragraph for each admissible pair $\left(T, T_{i}\right)$, obtaining an annular shell of tiles $\mathcal{F}_{j(i)}=\mathcal{F}_{j(i)}\left(T, T_{i}\right)$. For each admissible pair $\left(T, T_{i}\right)$, consider the collection of tiles $\mathcal{T}_{m}$ which are contained in $\mathcal{F}_{0} \cup \cdots \cup \mathcal{F}_{j(i)-1}$ but are not contained in $\mathcal{F}_{j\left(i^{\prime}\right)}\left(U, U_{i^{\prime}}\right)$ for any admissible pair $\left(U, U_{i^{\prime}}\right) \neq\left(T, T_{i}\right)$. Varying over all admissible pairs $\left(T, T_{i}\right)$, enumerate the resulting collections as $\mathcal{A}(m, j), 1 \leq j \leq N_{m}$. Define $\mathcal{B}(m, j)$ and $\mathcal{C}(m, j)$ by the conditions (5.4) and (5.5). Conditions (i), (ii), (iv) and (vi) follow directly from the construction. Conditions (iii) and (v) are consequences of (5.6). This completes the proof of the inductive step.

Proof of Proposition 5.2. Let $S \subset \mathbb{H}^{n}$ satisfy $\operatorname{dim}_{A} S<1$. In the first stage of the proof, we assume that $S$ is bounded. By a preliminary homothety, we may assume that $S \subset T_{o}$.

Choose $s \in\left(\operatorname{dim}_{A} S, 1\right)$. By Proposition 4.3, $S$ is $(b, k)$-sparse for some integer $b \geq b_{0}(s)$ and some $k \leq b^{s} \leq b /(500 n)$. Apply Lemma 5.4. 
For each $m$ and $j$, choose a tile $T_{(m, j)}=T\left(p_{(m, j)}, b^{m-1}\right) \in \mathcal{T}_{m-1}$ which is a child of one of the constituent tiles in $\mathcal{A}(m, j)$, and a tile $T^{(m, j)} \in \mathcal{T}_{m+1}$ so that $\cup \mathcal{B}(m, j) \subset \operatorname{star}\left(T^{(m, j)}\right)$. Define $u_{p_{(m, j)}}$ and $\left(F_{p_{(m, j)}, s}\right)$ as in Example 2.5. Our task is to calculate how large $s$ must be to guarantee that

$$
F_{p_{(m, j)}, s}\left(\operatorname{star}\left(T^{(m, j)}\right)\right) \subset T_{(m, j)} .
$$

Since

$$
\operatorname{star}\left(T^{(m, j)}\right) \subset B\left(p_{(m, j)}, \operatorname{diam}\left(\operatorname{star}\left(T^{(m, j)}\right)\right)\right) \subset B\left(p_{(m, j)}, 120 n b^{m+1}\right)
$$

and

$$
T_{(m, j)} \supset B\left(p_{(m, j)}, r_{i n} b^{m-1}\right),
$$

we see that (5.7) is satisfied provided

$$
120 n b^{m+1} e^{-s / 4} \leq r_{i n} b^{m-1} .
$$

The latter equation is satisfied when $s$ is equal to

$$
s_{0}:=4 \log \left(\frac{120 n b^{2}}{r_{i n}}\right) .
$$

Now, for fixed $m \leq 0$, set

$$
u_{m}=\sum_{j} \varphi_{(m, j)} u_{p_{(m, j)}},
$$

where $\varphi_{(m, j)} \in C_{0}^{\infty}\left(\mathbb{H}^{n}\right), 0 \leq \varphi_{(m, j)} \leq 1, \varphi_{(m, j)}=1$ on $\cup \mathcal{B}(m, j)$, and $\operatorname{supp} \varphi^{(m, j)} \subset$ $\cup \mathcal{C}(m, j)$. Denote by $\left(G_{m, s}\right)$ the corresponding flow of QC maps of $\mathbb{H}^{n}$. Choose $s=$ $s_{0}$ as above. One easily verifies that the maps $G_{m}:=G_{m, s_{0}}$ are all quasiconformal with uniformly bounded coefficient of quasiconformality. Note that $G_{m}$ acts as a (conformal) dilation in $\cup \mathcal{B}(m, j)$ for each $j$, and is equal to the identity on the complement of $\bigcup_{j} \cup \mathcal{C}(m, j)$. Moreover, $G_{m}$ takes $\cup \mathcal{B}(m, j)$ into $T_{(m, j)}$.

Now set $F_{m}=G_{m} \circ G_{m+1} \circ \cdots \circ G_{-1} \circ G_{0}$. By Lemma 5.4(iii), the maps $F_{m}$ are also all quasiconformal with uniformly bounded coefficient of quasiconformality, and equal to the identity in the complement of $T_{0}$. Furthermore, $F_{m}$ takes $\cup \mathcal{B}\left(m^{\prime}, j^{\prime}\right)$ into $T_{\left(m^{\prime}, j^{\prime}\right)}$ for each $m^{\prime} \in\{m, m+1, \ldots, 0\}$ and $j^{\prime}$.

By standard compactness theorems for quasiconformal maps, the maps $F_{m}$ converge pointwise to a limit map $F$ which is again quasiconformal. We check that the Assouad dimension of $S$ is decreased by $F$. By Lemma 5.4(vi), for each tile $T \in \mathcal{T}_{m}, m \leq 0, F(S) \cap T$ is contained in at most $k$ grandchildren of $T$, i.e., $F(S)$ is $\left(b^{2}, k\right)$-porous. By Proposition 4.3 .

$$
\operatorname{dim}_{A} F(S) \leq \frac{\log k}{\log b^{2}} \leq \frac{1}{2} s
$$

Thus $G C \operatorname{dim}_{A} S \leq \frac{1}{2} s$. Repeating $N$ times gives $G C \operatorname{dim}_{A} S \leq 2^{-N} s$. Letting $N \rightarrow \infty$ gives $G C \operatorname{dim}_{A} S=0$.

To remove the assumption that $S$ is bounded, we use the invariance of Assouad dimension under the inversion $j_{\mathbb{H}}$. Let $S$ be a subset of $\mathbb{H}^{n}$ with $\operatorname{dim}_{A} S<1$. We may assume that $S$ is closed by Proposition2.1(iii). Choose $p_{0} \notin S$ and $\epsilon>0$ so that $B_{\mathbb{H}}\left(p_{0}, \epsilon\right) \cap S=\emptyset$. Then $S^{\prime}=\left(j_{\mathbb{H}} \circ \delta_{1 / \epsilon}\right)\left(p_{0}^{-1} \cdot S\right)$ is bounded and $\operatorname{dim}_{A} S^{\prime}=\operatorname{dim}_{A} S$ by Proposition 2.6. The map $\Phi(p)=\left(j_{\mathbb{H}} \circ \delta_{1 / \epsilon}\right)\left(p_{0}^{-1} \cdot p\right)$ is 1-QC from $\mathbb{H}^{n} \backslash\left\{p_{0}\right\}$ to $\mathbb{H}^{n} \backslash\{o\}$ with $\lim _{p \rightarrow p_{0}}\|\Phi(p)\|_{\mathbb{H}}=+\infty$. If $F$ is a QC homeomorphism of $\mathbb{H}^{n}$, then $G:=j_{\mathbb{H}} \circ F \circ \Phi$ is a QC homeomorphism of $\mathbb{H}^{n} \backslash\left\{p_{0}\right\}$ to $\mathbb{H}^{n} \backslash\{o\}$ with 
$\lim _{p \rightarrow p_{0}}\|G(p)\|_{\mathbb{H}}=0$. Then $p_{0}$ is a removable singularity for $G$, and we may extend $G$ to a QC homeomorphism of $\mathbb{H}^{n}$. Since $\operatorname{dim}_{A} G(S)=\operatorname{dim}_{A} j_{\mathbb{H}}\left(F\left(S^{\prime}\right)\right)=$ $\operatorname{dim}_{A} F\left(S^{\prime}\right)$ we conclude that $G C \operatorname{dim}_{A} S^{\prime}=G C \operatorname{dim}_{A} S$. By the first part of the proof, $G C \operatorname{dim}_{A} S^{\prime}=0$. Hence also $G C \operatorname{dim}_{A} S=0$, which completes the proof.

Remark 5.5. Proposition 5.2 is best possible in the sense that there are sets of Assouad dimension one whose dimension cannot be lowered. However, restricting to subsets of the $t$-axis allows for a stronger statement, to wit,

$$
S \subset \mathbb{T}:=\left\{(z, t) \in \mathbb{H}^{n}: z=0\right\}, \operatorname{dim}_{A} S<2 \quad \Rightarrow \quad G C \operatorname{dim}_{A}^{\mathbb{H}^{n}} S=0 .
$$

Indeed, it is well known that the $\mathrm{CC}$ metric in $\mathbb{H}^{n}$ restricts to a multiple of $\sqrt{d_{E}}$ on T. By Proposition 2.1(vi), the condition $\operatorname{dim}_{A}\left(S, d_{c c}\right)<2$ for $S \subset \mathbb{T}$ translates to $\operatorname{dim}_{A}\left(S, d_{E}\right)<1$. By [46], there exist quasisymmetric self-maps $f$ of $\left(\mathbb{T}, d_{E}\right)$ so that $\operatorname{dim}_{A}\left(f(S), d_{E}\right)$ is arbitrarily small. Conjugating by the (quasisymmetric) snowflake map id $:\left(\mathbb{T}, d_{E}\right) \rightarrow\left(\mathbb{T}, d_{c c}\right)$ we may arrange that $f$ is a quasisymmetric self-map of $\left(T, d_{c c}\right)$. Moreover, the construction of these maps ensures that they may be extended as quasiconformal self-maps of $\mathbb{H}^{n} 6$ We conclude that $G C \operatorname{dim}_{A}^{\mathbb{H}^{n}} S=0$ as asserted. The corresponding result for Hausdorff dimension is likely to be true as well; one should check that the dimension-decreasing quasisymmetric maps constructed by Kovalev [33] in the case $V=\mathbb{R}$, after conjugating with the snowflake map, admit quasiconformal extensions to $\mathbb{H}^{n}$. It remains an open problem, to decide whether every quasisymmetric self-map of the $t$-axis admits a quasiconformal extension to $\mathbb{H}^{n}$. The precise value of the global (Hausdorff or Assouad) conformal dimension of $\mathbb{T}$ is also unknown. Heinonen and Semmes [27, Question 25] have asked whether $\mathbb{T}$ can be mapped onto a rectifiable curve by a quasiconformal selfmap of $\mathbb{H}^{1}$; the existence of such a map would of course imply that $G C \operatorname{dim}^{\mathbb{H}^{n}} \mathbb{T}$ is one. Balogh [4, Corollary 3.1] constructed subsets of $\mathbb{T}$ with Hausdorff dimension arbitrarily close to 2 which can be mapped into rectifiable curves by quasiconformal self-maps of $\mathbb{H}^{1}$.

Remark 5.6. In the Carnot groups which arise as local models for the Gromov boundaries of quaternionic hyperbolic space or the octonionic hyperbolic plane, all quasiconformal maps are 1-quasiconformal and smooth. (This observation combines Pansu's rigidity theorem [4] with the main result of Capogna and Cowling [17].) Thus

$$
G C \operatorname{dim}_{H} S=\operatorname{dim}_{H} S \text { and } G C \operatorname{dim}_{A} S=\operatorname{dim}_{A} S
$$

for every subset $S$ of one of these groups $\mathbb{G}$. For any $\alpha \in(0, Q)(Q$ the homogeneous dimension of $\mathbb{G}$ ) one can construct a self-similar subset $S$ of $\mathbb{G}$ which is Ahlfors regular of dimension $\alpha$, hence has $\operatorname{dim}_{A} S=\operatorname{dim}_{H} S=\alpha$. It follows that the analog of Theorem 1.1 is false in such groups, indeed, $G C \operatorname{dim}_{A}$ can take on any value in the interval $[0, Q]$.

Remark 5.7. Suppose $S \subset \mathbb{H}^{n}$ with $\operatorname{dim}_{H} S>0$. Is $\sup \operatorname{dim}_{H} F(S)=2 n+2$, where the supremum is taken over all QC maps $F: \mathbb{H}^{n} \rightarrow \mathbb{H}^{n}$ ? The analogous question in $\mathbb{R}^{n}$ has been answered in the affirmative by Bishop [9]. His proof relies on the Tukia-Väisälä extension theory for quasiconformal maps in $\mathbb{R}^{n}$ and admits no straightforward adaptation to $\mathbb{H}^{n}$. The analogous question for the Assouad dimension is open for both $\mathbb{H}^{n}$ and $\mathbb{R}^{n}$.

${ }^{6}$ We omit the details, which resemble those in the proof of Proposition 5.2 


\section{Appendix}

In this appendix, we present examples of Ahlfors $\alpha$-regular subsets of $\mathbb{H}^{n}$ for various $\alpha$. By Proposition 2.1(viii) each of these sets has Assouad and Hausdorff dimensions equal to $\alpha$.

Example 6.1. Let $S \subset \mathbb{H}^{n}$ be a set which is self-similar with respect to $D$, where $D$ is any of the metrics $d_{c c}, d$ or $d_{\mathbb{H}}$. In other words, we assume that there exist contractive similarity maps $F_{1}, \ldots, F_{M}$ of $\mathbb{H}^{n}$ so that

$$
S=F_{1}(S) \cup \cdots \cup F_{M}(S) \text {. }
$$

We denote by $r_{i}$ the contraction ratio for $F_{i}$, thus $D\left(F_{i}(p), F_{i}(q)\right)=r_{i} D(p, q)$ for all $p, q \in \mathbb{H}^{n}$. Examples include the Heisenberg cube and Strichartz tiles; further examples can be found in [5] and [8]. If the maps $\left\{F_{1}, \ldots, F_{M}\right\}$ satisfy the open set condition, then $S$ has positive and finite Hausdorff $\alpha$-measure, where $\alpha$ is the unique nonnegative solution to the equation

$$
\sum_{i=1}^{M} r_{i}^{\alpha}=1 .
$$

Thus $\operatorname{dim}_{H} S=\alpha$. In addition, the natural self-similar measure $\mu$ on $S$, characterized by the identity $\mu\left(F_{i_{1}} \circ \cdots \circ F_{i_{m}}(S)\right)=r_{1}^{\alpha} \cdots r_{m}^{\alpha}$ for all $\left(i_{1}, \ldots, i_{m}\right) \in$ $\{1, \ldots, M\}^{m}$, is Ahlfors $\alpha$-regular. This is part of the content of the MoranHutchinson theorem [37] and [28; see Kigami [30, section 1.5] for more details. By an appropriate choice of the $F_{i}$ we can arrange Cantor-type examples of this sort for each $\alpha \in[0,2 n+2]$.

Example 6.2. Let $S$ be any bounded $C^{1,1}$ hypersurface in $\mathbb{H}^{n}$ which bounds a domain $\Omega$. By Corollary 1.4 in 19 , the perimeter measure $P_{\mathbb{H}}(\Omega, \cdot)$ is an Ahlfors regular measure of dimension $2 n+1$ on $S$.

In the next example, we construct low codimensional Ahlfors regular subsets of $\mathbb{H}^{n}$ which are foliated by smooth hypersurfaces. In subsection 5.1, we show that these sets are minimal for global conformal Assouad dimension.

Recall that a point $p$ on a $C^{1}$ hypersurface $S \subset \mathbb{H}^{n}$ is called characteristic if $T_{p} S=H_{p} \mathbb{H}^{n}$. The characteristic locus of $S$ is the set of characteristic points. For $\epsilon>0$ we denote by

$$
P(S, \epsilon)=\left\{p \in \mathbb{H}^{n}: \operatorname{dist}_{c c}(p, S)=\epsilon\right\}
$$

the $\epsilon$-parallel set of $S$. When $S=\partial \Omega$, we also consider $P^{+}(S, \epsilon)=P(S, \epsilon) \cap\left(\mathbb{H}^{n} \backslash \bar{\Omega}\right)$, which is the boundary of the domain

$$
B(S, \epsilon)=\left\{p \in \mathbb{H}^{n}: \operatorname{dist}_{c c}(p, S)<\epsilon\right\} .
$$

Arcozzi and Ferrari [1] studied regularity properties of the distance function to a smooth hypersurface 7 We recall a special case of some of their results (see Theorems 1.1 and 5.2 and Corollary 5.1 in [1):

Proposition 6.3 (Arcozzi-Ferrari). Let $S$ be a noncharacteristic $C^{k}$ hypersurface in $\mathbb{H}^{n}$ which bounds a domain $\Omega$. Then

(i) $\operatorname{dist}_{c c}(\cdot, S)$ is of class $C^{k-1}$ in an open neighborhood of $S$.

\footnotetext{
${ }^{7}$ Note that the paper [1] treats only the case of the first Heisenberg group $\mathbb{H}^{1}$. The case of general $n$ will be treated in the forthcoming work [2].
} 
Furthermore, there exists $\epsilon>0$ so that

(ii) to every point $p \in B(S, \epsilon)$ there corresponds a unique point $p^{\prime} \in S$ with $d_{c c}\left(p, p^{\prime}\right)=d_{c c}(p, S)$, and

(iii) $p \mapsto\left(p^{\prime}\right.$, $\left.\operatorname{dist}_{c c}(p, S)\right)$ is a homeomorphism of $B(S, \epsilon) \backslash \bar{\Omega}$ onto $S \times(0, \epsilon)$.

It follows that $P^{+}(S, r)$ is a (bounded) $C^{k-1}$ hypersurface for $r<\epsilon$; if $k \geq 3$, then Example 6.2 implies that $P^{+}(S, r)$ is Ahlfors $(2 n+1)$-regular.

Remark 6.4. Hypersurfaces of the type described in Proposition 6.3 exist. For example, the standard torus $S=\left\{(z, t) \in \mathbb{H}^{n}:\left(|z|-r^{\prime}\right)^{2}+t^{2}=r^{2}\right\}, 0<r<r^{\prime}<\infty$, has empty characteristic locus. Indeed, the tangent hyperplane $T_{p_{0}} S$ at a point $p_{0}=\left(z_{0}, t_{0}\right) \in S$ is

$$
\left\{(z, t) \in \mathbb{R}^{2 n+1}:\left(1-\frac{r^{\prime}}{\left|z_{0}\right|}\right) \operatorname{Re}\left(z \overline{z_{0}}\right)+t_{0} t=0\right\}
$$

while the horizontal hyperplane $H_{p_{0}} \mathbb{H}^{n}$ is

$$
\left\{(z, t) \in \mathbb{R}^{2 n+1}: t+2 \operatorname{Im}\left(z \overline{z_{0}}\right)=0\right\} .
$$

If $T_{p_{0}} S=H_{0} \mathbb{H}^{n}$ we easily deduce that $\left(1-r^{\prime} /\left|z_{0}\right|\right)^{2}+4 t_{0}^{2}=0$, which is impossible for points $p_{0} \in S$.

Example 6.5. Let $S$ be a $C^{3}$ noncharacteristic hypersurface which bounds a domain in $\mathbb{H}^{n}$. Let $D \subset[0, \epsilon)$ be a standard (compact) Cantor set of (Euclidean) dimension $a, 0<a<1$, equipped with the natural Ahlfors $a$-regular measure $\mathcal{H}_{E}^{a}$. Define

$$
X:=\bigcup_{r \in D} P^{+}(S, r) .
$$

We claim that $X$ is Ahlfors regular of dimension $2 n+1+a$.

Define a measure $\mu$ on $X$ by

$$
\mu(A)=\int_{D} \mathcal{H}^{2 n+1}\left(A \cap P^{+}(S, r)\right) d \mathcal{H}_{E}^{a}(r) .
$$

By [20, Example I], this is the Method II measure constructed from the set function

$$
A \mapsto \int_{D}\left(\operatorname{diam} A \cap P^{+}(S, r)\right)^{2 n+1} d \mathcal{H}_{E}^{a}(r),
$$

in particular, it is a metric outer measure and hence a Borel measure. We verify the Ahlfors regularity condition

$$
\frac{1}{C} R^{2 n+1+a} \leq \mu\left(B\left(p_{0}, R\right) \cap X\right) \leq C R^{2 n+1+a}
$$

for all $p_{0} \in X$ and $0<R<\operatorname{diam} X$, for some fixed $C<\infty$.

The right hand inequality in (6.1) is easy. If $B\left(p_{0}, R\right) \cap P^{+}(S, r) \neq \emptyset$ for some $r \in D$, choose $p_{1} \in B\left(p_{0}, R\right) \cap P^{+}(S, r)$ and observe that

$$
B\left(p_{0}, R\right) \cap P^{+}(S, r) \subset B\left(p_{1}, 2 R\right) \cap P^{+}(S, r) .
$$

The result now follows from the Ahlfors $(2 n+1)$-regularity of $P^{+}(S, r)$, the Ahlfors $a$-regularity of $D$, and the definition of $\mu$.

To prove the left hand inequality in (6.1), let $p_{0} \in P^{+}\left(S, r_{0}\right)$ for some $r_{0} \in D$, let $0<R<\operatorname{diam} X$, and let $r_{1} \in D$ with $\left|r_{0}-r_{1}\right| \leq R / 2$. Set

$$
p_{1}=\mathcal{N}_{p_{0}}^{+}\left(P^{+}\left(S, r_{0}\right)\right)\left(r_{1}-r_{0}\right)
$$


where $\mathcal{N}_{p}^{+}(S)$ denotes the oriented metric normal to $S$ at $p$; see Definition 4.1 in [1. The fact which we require is the identity

$$
d_{c c}\left(\mathcal{N}_{q}^{+} F(t), q\right)=|t|,
$$

valid for any $C^{1}$ hypersurface $F, q \in F$ and $t$ sufficiently close to zero. In our situation, we obtain $p_{1} \in P^{+}\left(S, r_{1}\right)$ and an application of the triangle inequality and (6.2) gives $d_{c c}\left(p_{0}, p_{1}\right)=\left|r_{0}-r_{1}\right| \leq R / 2$. It follows that $B\left(p_{1}, \frac{1}{2} R\right) \cap P^{+}\left(S, r_{1}\right) \subset$ $B\left(p_{0}, R\right)$ for such $r_{1}$, and so

$$
\begin{aligned}
\mu\left(B\left(p_{0}, R\right)\right. & \cap X)=\int_{D} \mathcal{H}^{2 n+1}\left(B\left(p_{0}, R\right) \cap P^{+}(S, r)\right) d \mathcal{H}_{E}^{a}(r) \\
& \geq \int_{\left\{r_{1} \in D:\left|r_{0}-r_{1}\right| \leq R / 2\right\}} \mathcal{H}^{2 n+1}\left(B\left(p_{0}, R\right) \cap P^{+}(S, r)\right) d \mathcal{H}_{E}^{a}\left(r_{1}\right) \\
& \geq \int_{\left\{r_{1} \in D:\left|r_{0}-r_{1}\right| \leq R / 2\right\}} \mathcal{H}^{2 n+1}\left(B\left(p_{1}, \frac{1}{2} R\right) \cap P^{+}(S, r)\right) d \mathcal{H}_{E}^{a}\left(r_{1}\right) \\
& \geq C^{-1} R^{2 n+1} \mathcal{H}_{E}^{a}\left(\left\{r_{1} \in D:\left|r_{0}-r_{1}\right| \leq R / 2\right\}\right) \geq C^{-1} R^{2 n+1+a},
\end{aligned}
$$

where we used the $(2 n+1)$-regularity of $P^{+}(S, r)$, respectively the $a$-regularity of $D$, in the next-to-last, respectively last, line. Hence $X$ is $(2 n+1+a)$-regular.

\section{ACKNOWLEDGEMENTS}

I am grateful to Nicola Arcozzi for providing me with an early copy of [1] and discussing the forthcoming work [2].

\section{REFERENCES}

[1] Arcozzi, N., and Ferrari, F., Metric normal and distance function in the Heisenberg group. Math. Z. 256, 3 (2007), 661-684. MR2299576

[2] Arcozzi, N., Ferrari, F., and Montefalcone, F., in preparation.

[3] Assouad, P., Plongements lipschitziens dans $\mathbb{R}^{n}$. Bull. Soc. Math. France 111 (1983), 429448. MR763553 (86f:54050)

[4] BAlOGH, Z. M., Hausdorff dimension distribution of quasiconformal mappings on the Heisenberg group. J. Anal. Math. 83 (2001), 289-312. MR.1828495 (2002b:30022)

[5] Balogh, Z. M., Hoefer-Isenegger, R., And Tyson, J. T., Lifts of Lipschitz maps and horizontal fractals in the Heisenberg group. Ergodic Theory Dynam. Systems 26, 3 (2006). MR:2237461 (2007i:28009)

[6] Balogh, Z. M., AND TYson, J. T., Hausdorff dimensions of self-similar and self-affine fractals in the Heisenberg group. Proc. London Math. Soc. (3) 91, 1 (2005), 153-183. MR2149533 (2006b:28008)

[7] Balogh, Z. M., Tyson, J. T., And Warhurst, B., Gromov's dimension comparison problem on Carnot groups. C. R. Math. Acad. Sci. Paris 346, (2008), 135-138.

[8] Balogh, Z. M., Tyson, J. T., And Warhurst, B., Sub-Riemannian vs. Euclidean dimension comparison and fractal geometry on Carnot groups. Preprint, 2007.

[9] Bishop, C. J., Quasiconformal mappings which increase dimension. Ann. Acad. Sci. Fenn. Ser. A I Math. 24 (1999), 397-407. MR1724076 (2000i:30044)

[10] Bishop, C. J., And Tyson, J. T., Conformal dimension of the antenna set. Proc. Amer. Math. Soc. 129 (2001), 3631-3636. MR1860497(2002j:30021)

[11] Bishop, C. J., And Tyson, J. T., Locally minimal sets for conformal dimension. Ann. Acad. Sci. Fenn. Ser. A I Math. 26 (2001), 361-373. MR.1833245(2002c:30027)

[12] Bonk, M., And Kleiner, B., Conformal dimension and Gromov hyperbolic groups with 2sphere boundary. Geom. Topol. 9 (2005), 219-246 (electronic). MR.2116315 (2005k:20102)

[13] Bourdon, M., Au bord de certains polyèdres hyperboliques. Ann. Inst. Fourier (Grenoble) 45 (1995), 119-141. MR1324127(96b:20045) 
[14] Bourdon, M., Immeubles hyperboliques, dimension conforme et rigidité de Mostow. Geom. Funct. Anal. 7 (1997), 245-268. MR1445387 (98c:20056)

[15] Capogna, L., Regularity of quasilinear equations in the Heisenberg group. Comm. Pure Appl. Math. 50, 9 (1997), 867-889. MR1459590 (98k:22037)

[16] Capogna, L., Regularity for quasilinear equations and 1-quasiconformal maps in Carnot groups. Math. Ann. 313, 2 (1999), 263-295. MR1679786 (2000a:35027)

[17] Capogna, L., and Cowling, M., Conformality and $Q$-harmonicity in Carnot groups. Duke Math. J. 135, 3 (2006), 455-479. MR2272973 (2007h:30017)

[18] Capogna, L., Danielli, D., Pauls, S. D., and Tyson, J. T., An introduction to the Heisenberg group and the sub-Riemannian isoperimetric problem, vol. 259 of Progress in Mathematics. Birkhäuser Verlag, Basel, 2007. MR.2312336

[19] Capogna, L., and Garofalo, N., Ahlfors type estimates for perimeter measures in CarnotCarathéodory spaces. J. Geom. Anal. 16, 3 (2006), 455-497. MR2250055 (2008a:28005)

[20] Falconer, K. J., And Mauldin, R. D., Fubini-type theorems for general measure constructions. Mathematika 47 (2000), 251-265. MR1924502 (2003e:28009)

[21] Gehring, F. W., And VÄıs̈̈LÄ, J., Hausdorff dimension and quasiconformal mappings. J. London Math. Soc. (2) 6 (1973), 504-512. MR0324028 (48:2380)

[22] Gelbrich, G., Self-similar periodic tilings on the Heisenberg group. J. Lie Theory 4, 1 (1994), 31-37. MR1326950 (96e:22013)

[23] Heinonen, J., Calculus on Carnot groups. In Fall School in Analysis (Jyväskylä, 1994), vol. 68. Ber. Univ. Jyväskylä Math. Inst., Jyväskylä, 1995, pp. 1-31. MR.1351042 (96j:22015)

[24] Heinonen, J., A capacity estimate on Carnot groups. Bull. Sci. Math. 119 (1995), 475-484. MR:1354248 (96j:22011)

[25] Heinonen, J., Lectures on analysis on metric spaces. Springer-Verlag, New York, 2001. MR:1800917 (2002c:30028)

[26] Heinonen, J., And Koskela, P., Quasiconformal maps in metric spaces with controlled geometry. Acta Math. 181 (1998), 1-61. MR.1654771 (99j:30025)

[27] Heinonen, J., And Semmes, S., Thirty-three yes or no questions about mappings, measures, and metrics. Conform. Geom. Dyn. 1 (1997), 1-12. MR.1452413 (99h:28012)

[28] Hutchinson, J. E., Fractals and self-similarity. Indiana Univ. Math. J. 30 (1981), 713-747. MR.625600 (82h:49026)

[29] Keith, S., And LaAkso, T., Conformal Assouad dimension and modulus. Geom. Funct. Anal. 14, 6 (2004), 1278-1321. MR2135168 (2006g:30027)

[30] Kigami, J., Analysis on fractals, vol. 143 of Cambridge Tracts in Mathematics. Cambridge University Press, Cambridge, 2001. MR 1840042 (2002c:28015)

[31] Korányi, A., and Reimann, H. M., Quasiconformal mappings on the Heisenberg group. Invent. Math. 80 (1985), 309-338. MR788413 (86m:32035)

[32] Korányi, A., and Reimann, H. M., Foundations for the theory of quasiconformal mappings on the Heisenberg group. Adv. Math. 111 (1995), 1-87. MR.1317384 (96c:30021)

[33] Kovalev, L. V., Conformal dimension does not assume values between zero and one. Duke Math. J. 134, 1 (2006), 1-13. MR2239342 (2007c:51016)

[34] Lagarias, J. C., And Wang, Y., Self-affine tiles in $\mathbf{R}^{n}$. Adv. Math. 121, 1 (1996), 21-49. MR.1399601 (97d:52034)

[35] LuUkKainen, J., Assouad dimension: antifractal metrization, porous sets, and homogeneous measures. J. Korean Math. Soc. 35 (1998), 23-76. MR.1608518(99m:54023)

[36] Mattila, P., Geometry of sets and measures in Euclidean spaces, vol. 44 of Cambridge Studies in Advanced Mathematics. Cambridge University Press, Cambridge, 1995. MR1333890 (96h:28006)

[37] Moran, P. A. P., Additive functions of intervals and Hausdorff measure. Proc. Cambridge Philos. Soc. 42 (1946), 15-23. MR0014397 (7:278f)

[38] Mostow, G. D., Strong rigidity of locally symmetric spaces. Princeton University Press, Princeton, N.J., 1973. Annals of Mathematics Studies, No. 78. MR0385004 (52:5874)

[39] Pansu, P., Une inégalité isopérimétrique sur le groupe de Heisenberg. C. R. Acad. Sci. Paris Sér. I Math. 295, 2 (1982), 127-130. MR676380(85b:53044)

[40] Pansu, P., Dimension conforme et sphère à l'infini des variétés à courbure négative. Ann. Acad. Sci. Fenn. Ser. A I Math. 14 (1989), 177-212. MR.1024425 (90k:53079)

[41] PANSu, P., Métriques de Carnot-Carathéodory et quasiisométries des espaces symétriques de rang un. Ann. of Math. (2) 129 (1989), 1-60. MR979599 (90e:53058) 
[42] Strichartz, R. S., Self-similarity on nilpotent Lie groups. In Geometric analysis (Philadelphia, PA, 1991), vol. 140 of Contemp. Math. Amer. Math. Soc., Providence, RI, 1992, pp. 123-157. MR1197594 (94e:43011)

[43] Strichartz, R. S., Self-similarity in harmonic analysis. J. Fourier Anal. Appl. 1, 1 (1994), 1-37. MR 1307067 (96c:42002)

[44] Tyson, J. T., Quasiconformality and quasisymmetry in metric measure spaces. Ann. Acad. Sci. Fenn. Ser. A I Math. 23 (1998), 525-548. MR.1642158 (99i:30038)

[45] Tyson, J. T., Sets of minimal Hausdorff dimension for quasiconformal maps. Proc. Amer. Math. Soc. 128, 11 (2000), 3361-3367. MR.1676353 (2001b:30033)

[46] Tyson, J. T., Lowering the Assouad dimension by quasisymmetric mappings. Illinois J. Math. 45 (2001), 641-656. MR1878624 (2002k:30040)

[47] Trson, J. T., AND Wu, J.-M., Quasiconformal dimensions of self-similar fractals. Rev. Mat. Iberoamericana 22 (2006), 205-258. MR2268118(2008a:30024)

[48] VÄısÄLё, J., Porous sets and quasisymmetric maps. Trans. Amer. Math. Soc. 299 (1987), 525-533. MR869219 (88a:30049)

Department of Mathematics, University of Illinois, West Green Street, Urbana, ILLINOIS 61801

E-mail address: tyson@math.uiuc.edu 